\title{
A CHANDRA-SWIFT VIEW OF POINT SOURCES IN HICKSON COMPACT GROUPS: HIGH AGN FRACTION BUT A DEARTH OF STRONG AGNs
}

\author{
P. Tzanavaris ${ }^{1,2,7}$, S. C. Gallagher ${ }^{3}$, A. E. Hornschemeier ${ }^{1}$, K. Fedotov $^{3,6}$, M. Eracleous ${ }^{4,5}$, \\ W. N. Brandt ${ }^{4,5}$, T. D. Desjardins ${ }^{3}$, J. C. Charlton ${ }^{4}$, and C. Gronwall ${ }^{4,5}$ \\ ${ }^{1}$ Laboratory for X-ray Astrophysics, NASA/Goddard Spaceflight Center, Mail Code 662, Greenbelt, MD 20771, USA \\ ${ }^{2}$ Department of Physics and Astronomy, The Johns Hopkins University, Baltimore, MD 21218, USA \\ ${ }^{3}$ Department of Physics and Astronomy, The University of Western Ontario, London, ON N6A 3K7, Canada \\ ${ }_{5}^{4}$ Department of Astronomy and Astrophysics, The Pennsylvania State University, University Park, PA 16802, USA \\ ${ }^{5}$ The Institute for Gravitation and the Cosmos, The Pennsylvania State University, University Park, PA 16802, USA \\ ${ }^{6}$ Herzberg Institute of Astrophysics, Victoria, BC V9E 2E7, Canada \\ Received 2013 September 27; accepted 2014 March 5; published 2014 April 24
}

\begin{abstract}
We present Chandra X-ray point source catalogs for 9 Hickson Compact Groups (HCGs, 37 galaxies) at distances of 34-89 Mpc. We perform detailed X-ray point source detection and photometry and interpret the point source population by means of simulated hardness ratios. We thus estimate X-ray luminosities $\left(L_{X}\right)$ for all sources, most of which are too weak for reliable spectral fitting. For all sources, we provide catalogs with counts, count rates, powerlaw indices $(\Gamma)$, hardness ratios, and $L_{X}$, in the full $(0.5-8.0 \mathrm{keV})$, soft $(0.5-2.0 \mathrm{keV})$, and hard $(2.0-8.0 \mathrm{keV})$ bands. We use optical emission-line ratios from the literature to re-classify 24 galaxies as star-forming, accreting onto a supermassive black hole (AGNs), transition objects, or low-ionization nuclear emission regions. Two-thirds of our galaxies have nuclear X-ray sources with Swift/UVOT counterparts. Two nuclei have $L_{X, 0.5-8.0 \mathrm{keV}}>10^{42} \mathrm{erg} \mathrm{s}^{-1}$, are strong multi-wavelength active galactic nuclei (AGNs), and follow the known $\alpha_{\mathrm{OX}}-v L_{v \text { (nearUV) }}$ correlation for strong AGNs. Otherwise, most nuclei are X-ray faint, consistent with either a low-luminosity AGN or a nuclear $\mathrm{X}$-ray binary population, and fall in the "non-AGN locus" in $\alpha_{\mathrm{OX}}-v L_{v(\text { nearUV) }}$ space, which also hosts other normal galaxies. Our results suggest that HCG X-ray nuclei in high specific star formation rate spiral galaxies are likely dominated by star formation, while those with low specific star formation rates in earlier types likely harbor a weak AGN. The AGN fraction in HCG galaxies with $M_{R} \leqslant-20$ and $L_{X, 0.5-8.0 \mathrm{kev}} \geqslant 10^{41} \mathrm{erg} \mathrm{s}^{-1}$ is $0.08_{-0.01}^{+0.35}$, somewhat higher than the $\sim 5 \%$ fraction in galaxy clusters.
\end{abstract}

Key words: catalogs - galaxies: active - galaxies: nuclei - ultraviolet: galaxies - X-rays: galaxies

Online-only material: color figures, machine-readable tables

\section{INTRODUCTION}

By virtue of their selection criteria, Hickson Compact Groups (HCGs) constitute a distinct class among small galaxy agglomerations. The Hickson catalog (Hickson 1982; Hickson et al. 1992) comprises 92 spectroscopically confirmed nearby (median redshift $z_{\text {med }}=0.03, \sim 130 \mathrm{Mpc}$ ) compact groups with three or more members with accordant redshifts (i.e., within $1000 \mathrm{~km} \mathrm{~s}^{-1}$ of the group mean). The characteristic physical properties of CGs (Hickson et al. 1992) include galaxy separations of the order of a few galaxy radii (median projected separations $\sim 40 h^{-1} \mathrm{kpc}$ ), low velocity dispersions (radial median $\sim 200 \mathrm{~km} \mathrm{~s}^{-1}$ ), and high galaxy number densities (up to $10^{8} h^{2} \mathrm{Mpc}^{-2}$ ). These conditions favor galaxy interactions, as demonstrated by the spectacular examples of HCG 92 (Stephan's Quintet, e.g., Fedotov et al. 2011) and HCG 31 (Gallagher et al. 2010). It is thus natural to ask what influence this interaction-prone environment has on processes related to star formation or accretion onto a nuclear supermassive black hole.

With regard to star formation, recent work suggests that, compared to non-compact group environments, star formation is accelerated, leading to rapid exhaustion of the gas supply sustaining star forming activity. This result follows from ultraviolet and infrared star formation estimates that show significant discontinuities in mid-infrared colors and ultraviolet+infrared specific

\footnotetext{
7 NPP Fellow.
}

star formation rates (SSFRs; Johnson et al. 2007; Tzanavaris et al. 2010; Walker et al. 2010, 2012). In particular, the discontinuities indicate a bimodality between galaxies with high levels of star formation and those with little star formation. The latter have also been found to exhibit high levels of "H I deficiency," $D e f_{\mathrm{HI}}$, as defined by Verdes-Montenegro et al. (2001). These authors predict an expected $\mathrm{H}$ I mass for field galaxies of a given morphological type and compare it to the H I mass of compact group galaxies, thus calculating $D e f_{\mathrm{HI}}$. Taken together, the lack of galaxies with intermediate mid-infrared colors and SSFRs, as well as the high $D e f_{\mathrm{H}_{\mathrm{I}}}$ values are suggestive of accelerated then abruptly truncated star formation.

The importance of accretion onto a nuclear supermassive black hole (SMBH) in compact groups ("AGN"8) has not been thoroughly investigated and is not well established. In galaxy clusters Dressler et al. (1985) found fewer active galactic nuclei (AGNs) compared to the field (but see also Martini et al. 2006, and below). Compared to clusters, compact groups of galaxies have lower velocity dispersions, making prolonged close interactions more likely. It is thus possible that the level of AGN activity is different. On the theoretical and computational side, simulation work (e.g., Hopkins \& Quataert 2010) suggests that major galaxy mergers are a leading mechanism that can

\footnotetext{
8 In line with common usage in the literature we shall use the acronym "AGN" (active galactic nucleus) to refer to accretion onto a nuclear supermassive black hole. Strictly this is incorrect as nuclear activity can also be due to star formation.
} 
trigger inflow of rotationally supported gas to feed a central SMBH. Note, though, that this would also provide fuel for intense star formation and could trigger nuclear starbursts (e.g., Mihos \& Hernquist 1996). Other feeding mechanisms include supernova winds, minor interactions, and disk instabilities. Several observational surveys have provided insight on the connection between AGNs and galaxy interactions. For instance, Kartaltepe et al. (2010) find that AGNs are common in ultraluminous and hyperluminous infrared galaxies, which are known to result from major mergers. In addition, the AGN fraction in this population increases with infrared luminosity. Recently, Silverman et al. (2011) find increased AGN activity in pairs compared to isolated galaxies. On the other hand, several authors find minor interactions and secular evolution to be most important in triggering AGN activity (e.g., Grogin et al. 2005; Georgakakis et al. 2009; Cisternas et al. 2011; Deng et al. 2013).

In the optical regime, Coziol et al. (1998b, 1998a, 2004) used emission-line ratios in several samples (up to 91 galaxies in 27 compact groups) to determine the type of nuclear activity in compact group galaxies, consistently finding that strong and low-luminosity $\left(L_{\mathrm{H} \alpha} \lesssim 10^{39} \mathrm{erg} \mathrm{s}^{-1}\right.$ ) AGNs (LLAGNs) each make up no more than $\sim 10 \%$ of the total $C G$ populations (see Coziol et al. 2004, Table 3). Depending on the specific sample, star-forming galaxies represent a fraction of up to $\sim 34 \%$ of the population, with the remaining galaxies showing no emission lines. Both LLAGNs and AGNs are found mainly in optically luminous early type galaxies with little ongoing star formation that are in the centers of evolved groups. This finding was interpreted to indicate that such group cores are old, collapsed systems where star formation activity has ceased. According to this interpretation, high central densities of group cores induced gravitational interactions, which accelerated star formation, rapidly consuming all of the available fuel.

It is important to note that the fractions for "LLAGNs" presented by these authors also include low-ionization nuclear emission regions (LINERs), the nature of which is still a matter of debate. LINERs are characterized by high ratios of narrow optical low ionization oxygen emission lines (Heckman 1980) and are found in about half of all nearby galaxies (Ho et al. 1997). Candidate power sources for LINERs include (1) weak AGNs (e.g., Halpern \& Steiner 1983; Ferland \& Netzer 1983), (2) hot stars (e.g., Terlevich \& Melnick 1985; Filippenko \& Terlevich 1992; Shields 1992), and (3) shocks (e.g., Heckman 1980; Dopita et al. 1996). Although weak AGNs have been found in the majority $(\sim 75 \%)$ of LINERs (e.g., Barth et al. 1998; Ho et al. 2001; Filho et al. 2004; Nagar et al. 2005; Maoz et al. 2005; Flohic et al. 2006; González-Martín et al. 2009), they cannot account for the total LINER emission in the majority of cases (Eracleous et al. 2010a). In fact, for most LINERs Eracleous et al. (2010a) show that there is an energy deficit problem: star formation and AGN activity are not able to provide a sufficient number of ionizing photons to account for the observed emission lines.

In the most recent optical study, Martínez et al. (2010, hereafter M10) compiled a large spectroscopic sample of 280 galaxies in 64 HCGs and used emission-line ratios to classify the type of nuclear activity, providing an estimate for the AGN fraction in HCGs.

They classified $23 \%$ of galaxies as AGNs, $10 \%$ as transition objects (TO), and $14 \%$ as star forming (SF), with the remainder of the galaxies showing no emission lines. According to this study, although AGNs appear to be the most numerous emissionline galaxy class in CGs, they have characteristically low $\mathrm{H} \alpha$ luminosities (median $7.1 \times 10^{39} \mathrm{erg} \mathrm{s}^{-1}$ ) and virtually no broad emission lines, suggestive of LLAGNs. However, these authors use a restricted set of line ratios that precludes distinguishing between LINERs and AGNs.

In this paper we use the Kewley et al. (2006, hereafter K06) method to reclassify the galaxies of M10. This allows us to also identify LINER systems. To stress that this is an optically based classification, we use the designations optAGN, optTO, optSF, and optLINER.

Work in different wavelength regimes can provide complementary insight into these questions. Gallagher et al. (2008) used 1-24 $\mu \mathrm{m}$ 2MASS+Spitzer nuclear data to probe the nuclear activity in 46 galaxies from 12 nearby HCGs. They found that the spectral index, $\alpha_{\text {IRAC }}$, of a power law fit to the 4.5-8.0 $\mu \mathrm{m}$ IRAC data cleanly separates MIR-active from MIR-inactive HCG nuclei. Unfortunately, the exact origin of activity (whether AGN activity or star formation) cannot be deduced by this method. In particular, these authors show that hot dust emission can be responsible for their results, and this can be due either to hard ionizing AGN continua or asymptotic giant branch populations in star-forming galaxies. On the other hand, Roche et al. (1991) have shown that MIR-inactivity $\left(\alpha_{\text {IRAC }}>0\right)$ is associated with low-luminosity AGN activity.

Due to the high-energy emission generated by supermassive black hole accretion, by far the best direct diagnostic for strong AGN activity is nuclear X-ray emission. Compared to the optical, the X-ray regime offers the advantage that the nuclear emission is not diluted by starlight from the host galaxy, while dust obscuration is very significantly mitigated due to the higher, penetrating power of X-ray radiation. Unfortunately, this simple picture is complicated by the combined effect of two factors. First, X-ray starlight can sometimes dilute AGN emission. This is because X-ray binary (XRB) populations in circumnuclear star clusters also emit in the X-ray regime, although individual XRBs typically have lower luminosities than strong AGNs. Second, as the name implies, LLAGNs emit at low X-ray luminosities. Adopting a fiducial threshold of $L_{X, 0.5-8.0 \mathrm{keV}}=10^{41-42} \mathrm{erg} \mathrm{s}^{-1}$, it is only at higher X-ray luminosities that nuclear X-ray emission can be attributed to an AGN with high probability. Thus the situation becomes increasingly ambiguous at progressively fainter luminosities, making it challenging to distinguish between X-ray emission due to unresolved populations of circumnuclear XRBs and that of LLAGNs. In this regime high angular resolution becomes critical for distinguishing nuclear from circumnuclear emission.

Although authors of earlier studies detected X-ray emission in HCGs, they were hampered by poor angular resolution and the lack of hard X-ray sensitivity, making it difficult to disentangle the contributions from point source (nuclear or extra-nuclear) and diffuse emission; they essentially concentrated on studying the diffuse component. Using ROSAT data, Ponman et al. (1996) detected diffuse intergalactic medium (IGM) in $\sim 75 \%$ of a large HCG sample, while Mulchaey et al. (2003), using a low-redshift sample of 109 groups that included poor compact as well as rich non-compact systems found diffuse, extended $\mathrm{X}$-ray emission in 61 groups (56\%). In an effort to understand the relevance of ram-pressure stripping and strangulation due to hot IGM in the most H I-deficient HCGs, Rasmussen et al. (2008) also examined the level of nuclear activity in a sample of eight HCGs, finding no significant enhancement. However, they do not carry out a detailed high angular resolution study to provide more specific results on the nature of nuclear activity in their systems. 
Table 1

Chandra Observation Log for This HCG Sample

\begin{tabular}{|c|c|c|c|c|c|c|c|}
\hline $\begin{array}{l}\text { HCG ID } \\
\text { (1) }\end{array}$ & $\begin{array}{l}\text { Obs. ID } \\
\text { (2) }\end{array}$ & $\begin{array}{c}\text { Obs. Start Date } \\
\text { (3) }\end{array}$ & $\begin{array}{l}\text { Detector } \\
\text { (4) }\end{array}$ & $\begin{array}{l}\text { Obs. Time }(\mathrm{ks}) \\
(5)\end{array}$ & $\begin{array}{l}\text { Obs. Type } \\
\text { (6) }\end{array}$ & $\begin{array}{l}\text { PI } \\
\text { (7) }\end{array}$ & $\begin{array}{l}\text { References } \\
\text { (8) }\end{array}$ \\
\hline HCG 7 & 8171 & 2007 Sep 13 & ACIS-S & 19.4 & GTO & Garmire & \\
\hline \multirow[t]{2}{*}{ HCG 7} & 9588 & 2007 Sep 16 & ACIS-S & 16.9 & GTO & Garmire & \\
\hline & & & & 36.3 & & & Konstantopoulos et al. (2010) \\
\hline HCG 16 & 923 & 2000 Nov 16 & ACIS-S & 12.7 & GO & Mamon & Jeltema et al. (2008) \\
\hline HCG 22 & 8172 & 2006 Nov 23 & ACIS-S & 32.2 & GTO & Garmire & Desjardins et al. (2013) \\
\hline HCG 31 & 9405 & 2007 Nov 15 & ACIS-S & 36.0 & GO & Gallagher & Smith et al. (2012) \\
\hline HCG 42 & 3215 & 2002 Mar 26 & ACIS-S & 32.1 & GO & Ponman & Jeltema et al. (2008) \\
\hline HCG 59 & 9406 & 2008 Apr 12 & ACIS-S & 38.9 & GO & Gallagher & Desjardins et al. (2013) \\
\hline HCG 62 & 921 & $2000 \mathrm{Jan} 25$ & ACIS-S & 49.1 & GO & Vrtilek & \\
\hline HCG 62 & 10462 & 2009 Mar 2 & ACIS-S & 68.0 & GO & Rafferty & \\
\hline \multirow[t]{2}{*}{ HCG 62} & 10874 & 2009 Mar 3 & ACIS-S & 52.0 & GO & Rafferty & \\
\hline & & & & 169.2 & & & Jeltema et al. (2008) \\
\hline HCG 90 & 905 & $2000 \mathrm{Jul} 2$ & ACIS-I & 50.2 & GO & Bothun & Jeltema et al. (2008) \\
\hline HCG 92 & 7924 & 2007 Aug 17 & ACIS-S & 94.4 & GO & Vrtilek & \\
\hline \multirow[t]{2}{*}{ HCG 92} & 789 & $2000 \mathrm{Jul} 9$ & ACIS-S & 20.0 & GO & Trinchieri & \\
\hline & & & & 114.4 & & & O’Sullivan et al. (2009) \\
\hline
\end{tabular}

Notes. Columns are: 1: HCG group name; 2: observation ID; 3: start date of observation; 4: detector; 5: exposure time; 6: observation type (Guaranteed Observing Time or General Observer proposal); 7: principal investigator; 8: references (first publication using these data). Total exposure times for each group appear in bold.

The level of AGN activity in galaxy clusters has already been systematically investigated in the X-ray regime, leading to differing conclusions (e.g., see Ehlert et al. 2013 for a review). Using a multi-wavelength approach that includes emission lines, $\mathrm{X}$-ray spectral properties, and $\mathrm{X}$-ray to visible-wavelength flux ratios in rich clusters, Martini et al. (2006) found that $\sim 5 \%$ of cluster galaxies more luminous than $M_{R}=-20$ host AGNs with $L_{X, 0.5-8.0 \mathrm{keV}}>10^{41} \mathrm{erg} \mathrm{s}^{-1}$. They also notably found a discrepancy between the AGN fraction determined from optical spectroscopy and a higher fraction suggested by X-ray luminosities. Interestingly, Shen et al. (2007) compared the environments of poor groups and clusters using a combined optical and X-ray approach. They concluded that poor groups host AGNs that are in an optically dominant phase, whereas those in clusters are dominant in the X-rays, leading to the findings of Martini et al. (2006).

To date, there has been no systematic study of nuclear X-ray emission in compact groups. In this paper we take advantage of the superb angular resolution of the Chandra X-ray observatory to carry out a detailed point source detection in a sample of 9 compact groups (37 galaxies). This paper has two main goals. First, we make available full X-ray source catalogs based on the Chandra observations in nine compact group fields with detailed information on counts, fluxes, luminosities, and hardness ratios. Second, we focus on point sources located in HCG galaxy nuclei. Using Chandra and Swift/Ultra-Violet and Optical Telescope (UVOT; Roming et al. 2005) data, we combine X-ray and ultraviolet (UV) nuclear photometry, and compare this with radio and optical diagnostics to assess the nature of nuclear activity in compact group galaxies. In a separate paper, we discuss the diffuse X-ray emission in the same sample of compact groups (Desjardins et al. 2013). Some of the Chandra data have been previously presented in a different context. We give appropriate references in Table 1.

The structure of the paper is as follows. Section 2 introduces our sample. Section 3 discusses X-ray data and analysis and point source detections. Section 4 presents UV nuclear data and analysis. Section 5 presents multi-wavelength analyses, including new optical emission-line ratio classifications, radio data, and a combined X-ray -UV analysis. Section 6 presents estimates on the AGN fraction in HCGs and Section 7 discusses our findings. We conclude with a summary in Section 8.

\section{SAMPLE SELECTION}

Our original multi-wavelength HCG sample comprises 11 groups compiled from the original HCG catalog of 92 spectroscopically confirmed compact groups (Hickson et al. 1992). This has been the most widely used ("benchmark," Lee et al. 2004) of all CG catalogs. Although valid concerns about this catalog have been raised regarding selection biases (e.g., Mamon 1994; Ribeiro et al. 1998), comparisons with recent larger catalogs show that many HCG galaxy properties such as surface brightness or angular and linear diameter are in fact close to median values for the corresponding distributions (Lee et al. 2004).

In order to ensure that our sample would be observable with a range of ground- and space-based instruments for our longterm multi-wavelength campaign, the selection was based on membership (a minimum of three giant galaxies with accordant redshifts, i.e., within $1000 \mathrm{~km} \mathrm{~s}^{-1}$ of the group mean), distance $\left(\lesssim 4500 \mathrm{~km} \mathrm{~s}^{-1}\right)$, and angular extent $\left(\lesssim 8^{\prime}\right.$ in diameter).

In this paper we present nine of these groups, for which both archival Chandra X-ray and Swift UVOT ultraviolet data are available. Swift/UVOT false color images of the group fields, with detected Chandra X-ray point sources overlaid are shown in Figure 1. An observation log for the Chandra data is presented in Table 1. The Chandra observations include Guaranteed Observing Time (HCGs 7 and 22, PI: Garmire). An observation $\log$ for the Swift UVOT data is presented in Tzanavaris et al. (2010). In addition, note that in the present work we have included UVOT data for HCGs 90 and 92 (see below). The group and galaxy IDs, as well as morphological types, can be found in the first two columns of Table 11, which also provides an overview of our multi-wavelength results (Section 5).

As the 9 groups used in this paper represent a small archival sub-sample of the full set of 92 compact groups, we do not a priori expect them to be fully representative of all HCGs. 

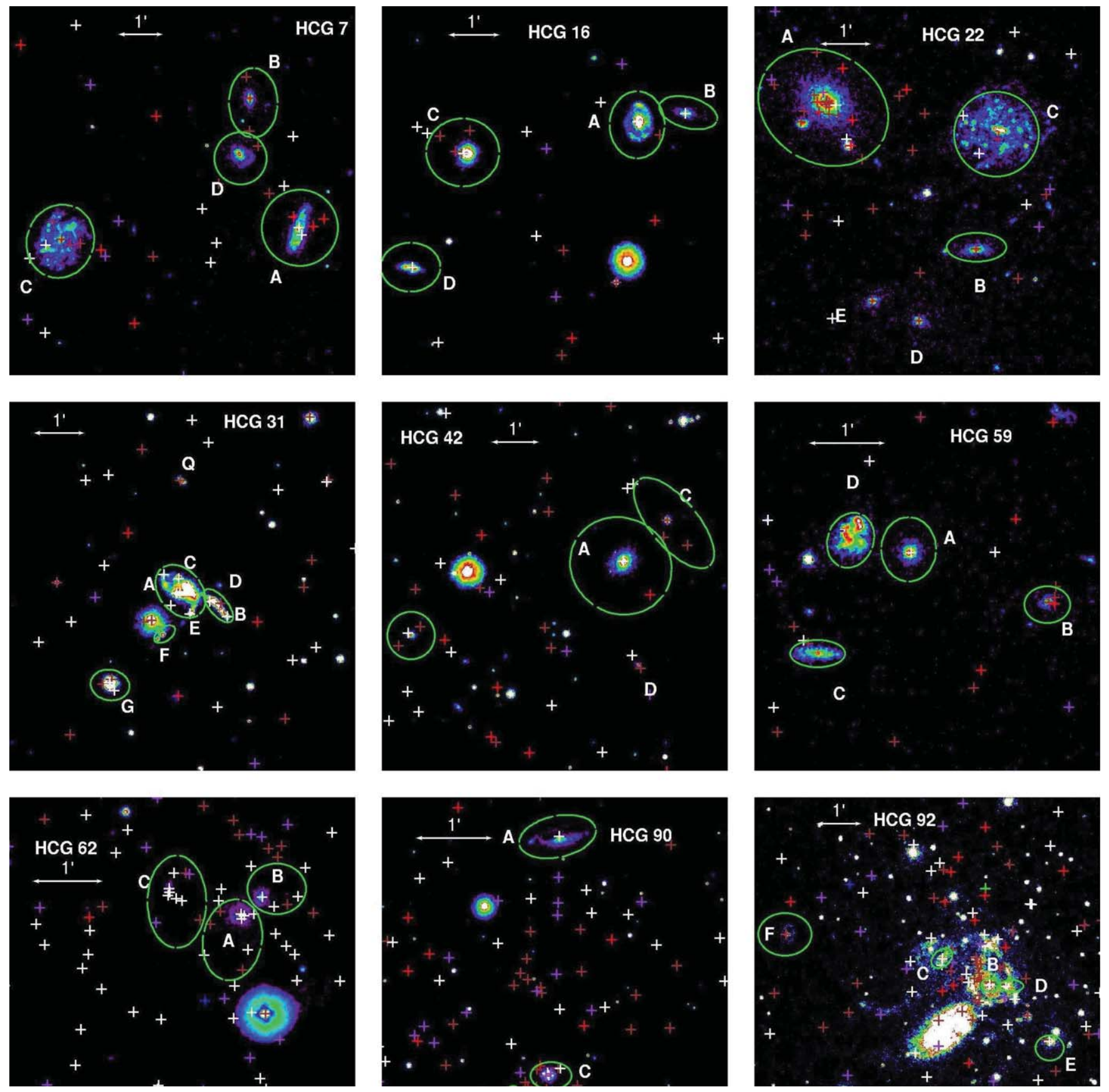

Figure 1. Swift-UVOT false color $u v w 1$ images with Chandra X-ray point sources overlaid. UV intensity increases from violet (lowest) to blue to green to red to white (highest). The color coding of source symbols indicates whether they are detected in the full band only (red); the soft band only (green); the hard band only (blue); the full and the hard bands (purple); the full and the soft bands (brown); the soft and the hard (blue-green) bands; or the full, soft, and hard bands (white). The green ellipses define galaxy regions from the mid-infrared work of Johnson et al. (2007). The bright source to the right of the center in the HCG 62 field is a foreground star. (A color version of this figure is available in the online journal.)

We further investigate this issue by comparing the distributions of a number of characteristic properties, established and tabulated by Hickson (1982) and Hickson et al. (1992), for both the full set and our sub-sample. These include the number of galaxies per group, $n$, the radial velocity dispersion, $\sigma_{v}$, the median projected separation, $D$, and the angular diameter, $\theta_{G}$. The distributions of these properties for both the full sample and our nine-group subsample our shown in Figure 2. The distribution for $n$ is very similar for the two populations, with means of $\left\langle n_{92}\right\rangle=4.2 \pm 1.0$ and $\left\langle n_{9}\right\rangle=3.8 \pm 0.4$ for the 92 and 9 HCG samples, respectively. A Kolmogorov-Smirnov (KS) test gives a modestly high probability of 0.005 that the two distributions come from the same parent population. The situation for $D$ is similar $\left(\left\langle D_{92}\right\rangle=(1.6 \pm 0.3) \mathrm{kpc}\right.$ and $\left.\left\langle D_{9}\right\rangle=(1.4 \pm 0.2) \mathrm{kpc}\right)$, with an even higher KS probability (0.2). The $\sigma_{v}$ distributions are less similar in terms of their peaks but their means are fully consistent within the uncertainties $\left(\left\langle\sigma_{v, 92}\right\rangle=(225 \pm 30) \mathrm{km} \mathrm{s}^{-1}\right.$ and $\left.\left\langle\sigma_{v, 9}\right\rangle=(212 \pm 31) \mathrm{km} \mathrm{s}^{-1}\right)$. However, the KS probability that the distributions are the same is relatively high (0.3). Finally, the distributions for $\theta_{G}$ are also less similar, and are consistent within $2 \sigma\left(\left\langle\theta_{G, 92}\right\rangle=(3.7 \pm 2.8)^{\prime}\right.$ and $\left.\left\langle\theta_{G, 9}\right\rangle=(4.5 \pm 2.2)^{\prime}\right)$. This is to be expected, as our selection criteria are biased toward 

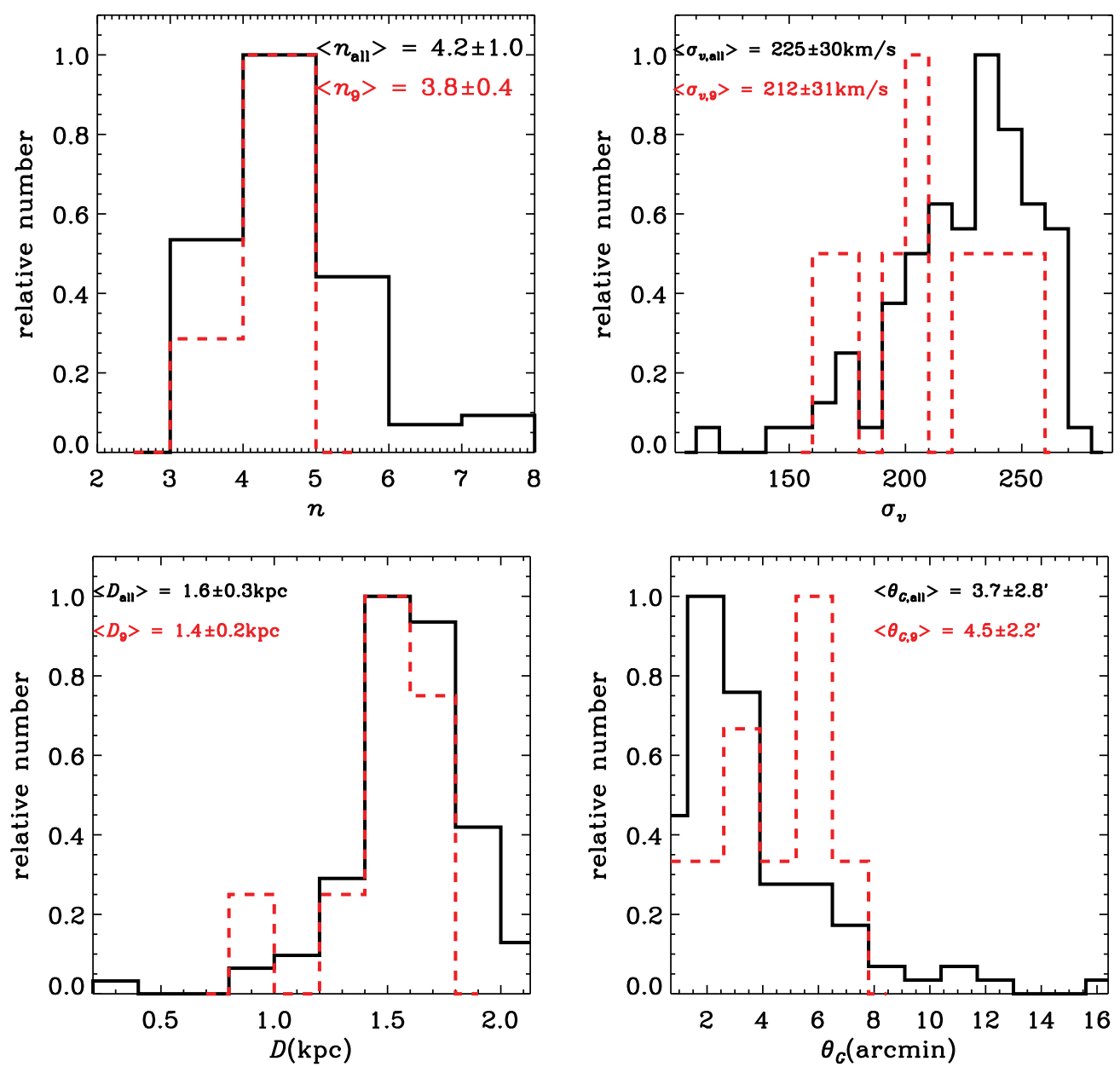

Figure 2. Histograms for physical properties of the 9 galaxy groups in this paper (red, dashed lines) and the full sample of 92 Hickson Compact Groups (Hickson 1982; Hickson et al. 1992, black continuous lines). Clockwise from top left, properties shown are the mean number of galaxies per group with accordant redshifts, the mean radial velocity dispersion of galaxies per group, the mean projected separation, and the mean angular size of galaxies per group. Mean values and standard deviations are shown in the upper right corner of each panel.

(A color version of this figure is available in the online journal.)

more nearby systems. Again, the KS probability is relatively high (0.3). Thus, overall, we find that the sub-sample used in this paper is reasonably representative of HCGs as a class.

\section{X-RAY DATA ANALYSIS}

\subsection{Point Source Detection and Photometry}

Each group was observed at the aim point of the backilluminated S3 CCD of Chandra's Advanced CCD Imaging Spectrometer (ACIS), with the exception of HCG 90, which was observed with the ACIS-I array. The data were processed using standard Chandra X-ray Center aspect solution and grade filtering, from which the level 2 events file was produced. Due to Chandra's $\sim 1^{\prime \prime}$ angular resolution and the proximity of our galaxies, we can detect a multitude of individual point sources in our fields. We have thus been able to carry out a detailed point source detection and characterization consisting of four stages as follows.

First, the CIAO 4.1.2 9 wavelet detection tool WAVDETECT (Freeman et al. 2002) was used in the soft $(S, 0.5-2.0 \mathrm{keV})$, hard $(H, 2.0-8.0 \mathrm{keV})$, and full $(F, 0.5-8.0 \mathrm{keV})$ bands to detect candidate point sources in each band. The lower limit of $0.5 \mathrm{keV}$

\footnotetext{
9 http://cxc.harvard.edu/ciao
}

matches the well-calibrated part of the response, while above $8.0 \mathrm{keV}$ the effective area of the Chandra mirrors is known to drop considerably and the particle background increases significantly. The chip field $(1024 \times 1024$ pixels for S3 and $2048 \times 2048$ pixels for I0-I3) was searched with WAVDETECT at the $10^{-5}$ false-probability threshold in all three energy bands. Although a lower probability threshold $\left(10^{-6}\right.$ per CCD) is often used to ensure low false positive detections, the situation is more complicated for false negatives, especially near an observation's detection limit (Kim et al. 2004). We thus chose to detect a greater number of spurious sources at this stage, which were excluded at subsequent stages of the analysis as explained below. For comparison, we show the numbers of sources detected with WAVDETECT using these two probability thresholds for each HCG field in the three X-ray bands in Figure 3. Wavelet scales used were $1,1.414,2,2.828,4,5.657$, and 8.0 pixels to cover a wide variety of source sizes, as well as to take into account the variation of the point-spread function (PSF) size across the ACIS CCD. Source lists produced by WAVDETECT for each band were cross-correlated to produce a single list of positions for candidate point sources in each field. This matching used each source's PSF ellipses, whose size and orientation depend on the detector used (ACIS-S or I), the position on the detector, and the roll angle of observations. 


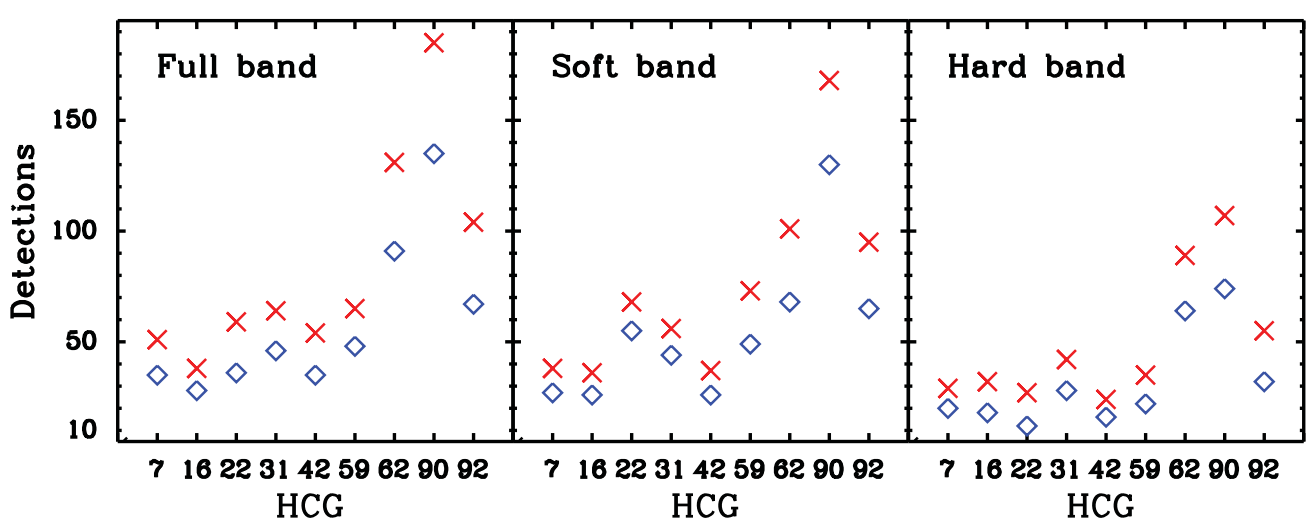

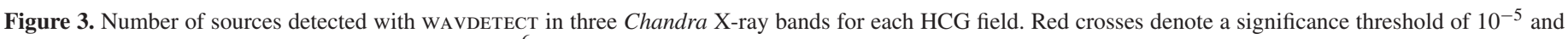
blue diamonds indicate a significance threshold of $10^{-6}$.

(A color version of this figure is available in the online journal.)

The second stage of the point source analysis involved using the software ACIS EXTRACT (AE, Broos et al. 2010, ${ }^{10}$ ) to perform aperture photometry for sources in the WAVDETECT source list. Some of the features of AE that make it a good choice for ACIS point source extraction include the following.

1. Construction of PSF-shaped aperture extraction regions for each source and each observation separately. These regions encircle $\sim 90 \%-60 \%$ of the photon energy at $1.5 \mathrm{keV}$, depending on how crowded a given field region is.

2. Construction of background regions that exclude pixels from neighboring sources and, where appropriate, a model of the wings of a neighboring source's contaminating emission.

3. Use of the Chandra Calibration Database for producing ancillary reference files (ARFs) and response matrix files (RMFs) for each source and observation, appropriately merging these for multiple observations.

4. Aperture corrections by means of calculation of the energy fraction falling inside the PSF region at five different energies.

Our initial catalogs included all sources detected at the AE stage. We then flagged sources with negative net (source background) counts in a given band as non-detections in that band. Such sources were assigned the detection flag 1 in that band. Further, we obtained Poisson $\pm 1 \sigma$ errors on net counts by using the method of Gehrels (1986). If the measured net counts minus the lower $2 \sigma$ error thus calculated were $\leqslant 0$, sources were also flagged as non-detections. To distinguish these from the previous type of non-detections, these were assigned the detection flag 5 in that band. For non-detections we estimated upper confidence limits. ${ }^{11}$ for fluxes and confidence levels $\mathrm{CL}=0.90$ by following Kraft et al. (1991). In this approach, the probability that a source flux, $S$, lies between $S_{\min }$ and $S_{\max }$ is given by

$$
\mathrm{CL}=\int_{S_{\min }}^{S_{\max }} f_{N, B}(S) d S
$$

where the posterior probability function for parameter $S$ as a function of the observed counts $N$ and the mean background $B$

\footnotetext{
10 Package and User's Guide available at http://www.astro.psu.edu/xray/ acis/acis_analysis.html

11 We use the term upper confidence limit to stress that this is an estimate of the upper edge of a confidence interval for the source intensity regardless of the detection procedure. The term upper limit should be reserved for characterizing the detection process (Kashyap et al. 2010).
}

is given by

$$
f_{N, B}(S)=C \frac{e^{-(S+B)}(S+B)^{N}}{N !}
$$

and $C$ is a normalization constant (Equation (8) in Kraft et al. (1991).)

In our catalogs, we also include alternative detection criteria (see below).

Most of our sources have few net counts, precluding reliable spectral fitting. Thus at the third and final stage of the point source analysis, we applied the method of Gallagher et al. (2005), which makes use of hardness ratios to obtain rough estimates of spectral shapes and, hence, fluxes and luminosities for each source. The hardness ratio is defined as

$$
\mathrm{HR} \equiv(H-S) /(H+S)
$$

where $H$ and $S$ represent net counts in the hard and soft bands, respectively. For each source this method compares the observed hardness ratio to that obtained from simulated spectra in order to estimate the power-law index $\Gamma$ (where the photon flux is given by $f_{E} \propto E^{-\Gamma}$ photon $\mathrm{cm}^{-2} \mathrm{~s}^{-1} \mathrm{keV}^{-1}$ ) and associated X-ray flux and luminosity. We used the X-ray spectral modeling tool XSPEC (Arnaud 1996), version 12.5.0, to construct grids of simulated spectra. For each source, we used the corresponding Galactic column density, $N_{\mathrm{H}}^{\mathrm{gal}}$, as well as the ARFs and RMFs produced by AE. We imposed a simple absorbed power law model (tbabs*po in XSPEC) and varied $\Gamma$ in the range $-1 \rightarrow+4$ to obtain simulated count rates in the full, soft, and hard bands and thus simulated HR values. By comparing these values with the observed HR, we estimated the best $\Gamma$ values and corresponding fluxes and luminosities. We illustrate this process in Figure 4. Each panel corresponds to a single X-ray HCG field. For each source detected in at least one band in this field we plot the simulated HR values against corresponding $\Gamma$ values. Thus, each gray curve in a panel is made up of a set of simulated $\mathrm{HR}-\Gamma$ pairs for a single detected source. Given an observed $\mathrm{HR}$ value and a simulated $\mathrm{HR}-\Gamma$ curve, there is then a unique $\Gamma$ value that provides the best observed $\Gamma$ estimate, as indicated by the blue triangles. ${ }^{12}$

Due to the simplicity of our model, which assumes no intrinsic absorption $\left(N_{\mathrm{H}}^{\text {int }}=0\right)$, it is likely that some $\Gamma$ values are incorrect. However, $\Gamma$ and $N_{\mathrm{H}}$ are degenerate, so this should have a minimal

12 We stress that there is only one blue triangle per curve, although the high density of curves in Figure 4 may suggest otherwise. 


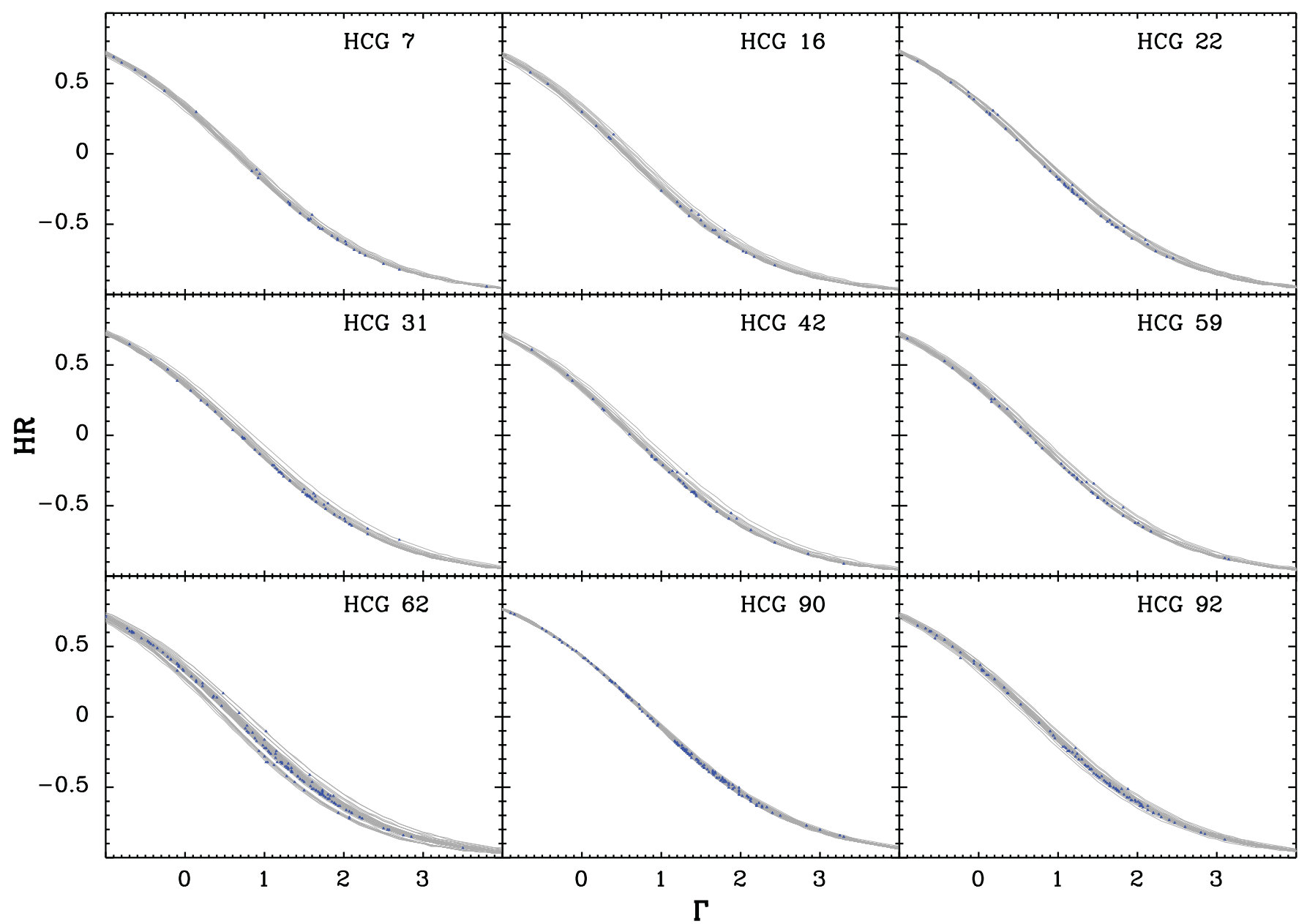

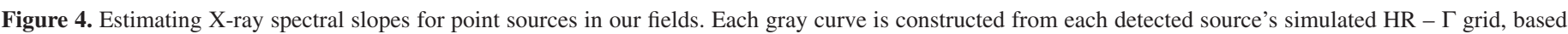

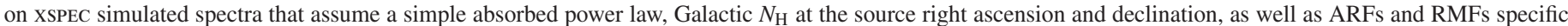

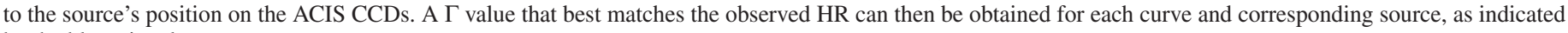
by the blue triangles.

(A color version of this figure is available in the online journal.)

effect on luminosity, which is the quantity in which we are most interested. As a comparison, for the nucleus of HCG 62 A our method yields luminosities $L_{X, 2.0-8.0 \mathrm{keV}}=1.0 \times 10^{39} \mathrm{erg} \mathrm{s}^{-1}$ and $L_{X, 0.5-8.0 \mathrm{keV}}=3.2 \times 10^{39} \mathrm{erg} \mathrm{s}^{-1}$. These are in good agreement with the results reported by Rafferty et al. (2013). Using the same Chandra data, these authors carried out more detailed spectral fitting that included both intrinsic and Galactic absorption as well as a thermal component and they reported $L_{X, 2.0-10.0 \mathrm{keV}}=(1.1 \pm 0.4) \times 10^{39} \mathrm{erg} \mathrm{s}^{-1}$ and $L_{X, 0.5-7.0 \mathrm{keV}}=$ $1.5_{-1.0}^{+2.8} \times 10^{39} \mathrm{erg} \mathrm{s}^{-1}$.

Point source catalogs for all sources are presented in Table 2. For completeness, the tables include sources considered both detected and undetected, according to our conservative $2 \sigma$ criterion above. We have compiled two sets of tables. The first set (Table 2) presents $\Gamma$ values, fluxes, and luminosities in the full, soft, and hard X-ray bands, derived as explained above, as well as the flux density at $2 \mathrm{keV}$.

The second set of tables (Table 3 ) presents counts and count rates in the full, soft, and hard bands.

Apart from the Gehrels detection criterion, for completeness in this second set of tables we also include the two AE detection criteria, namely the AE significance, which is essentially a traditional signal-to-noise criterion, as well as the binomial probability, $P_{B}$, that a source is spurious (Equation (4) below; see Broos et al. 2010, Appendix B for details). Users of our catalogs are left to choose which detection criterion they prefer.

In both sets of tables, we also include two types of detection/ non-detection flags for each source. As already mentioned, the first type of flag (columns 17, 18, 19 in Table 2; columns 3, 5,7 in Table 3) is related to the relative numbers of source and background counts, and is equal to 1 (net counts negative; no detection in band), 5 (net counts minus Gehrels $2 \sigma$ error $<0$ ), or 0 (unambiguous detection in band). The second type of flag (column 16 in Table 2 and column 9 in Table 3) indicates whether the hardness ratio is an upper or lower limit. From the HR definition (Equation (3)) it follows that if there is a detection in the hard band but not the soft band, an HR value is a lower limit (flag value equal to -1). Conversely, if there is a detection in the soft band but not in the hard band, an HR value is an upper limit (flag value equal to 1 ). If there is no detection in either band, this flag is equal to -2 , and if there is a detection in both bands, the flag is equal to 0 .

Finally, in both sets of tables, for sources that fall within the boundaries of individual galaxies (defined as explained in Section 3.2) an uppercase letter in column 1 (ID) indicates the galaxy to which they belong. If these are also nuclear sources, this is indicated by an asterisk next to the galaxy's letter designation. 
Table 2

Derived Quantities and Detection Codes for X-Ray Point Sources in HCG 7

\begin{tabular}{|c|c|c|c|c|c|c|c|c|c|c|c|c|c|c|c|c|c|c|c|c|c|}
\hline \multirow[b]{2}{*}{$\begin{array}{l}\text { ID } \\
\text { (1) }\end{array}$} & \multirow[b]{2}{*}{$\begin{array}{c}\Gamma \\
(2)\end{array}$} & \multirow[b]{2}{*}{$\begin{array}{c}+ \\
(3)\end{array}$} & \multirow[b]{2}{*}{$\begin{array}{l}- \\
(4)\end{array}$} & \multirow[b]{2}{*}{$\begin{array}{l}f_{F} \\
(5)\end{array}$} & \multirow[b]{2}{*}{$\begin{array}{l} \pm \\
(6)\end{array}$} & \multirow[b]{2}{*}{$\begin{array}{l}f_{S} \\
(7)\end{array}$} & \multirow[b]{2}{*}{$\begin{array}{c} \pm \\
(8)\end{array}$} & \multirow[b]{2}{*}{$\begin{array}{l}f_{H} \\
(9)\end{array}$} & \multirow[b]{2}{*}{$\begin{array}{c} \pm \\
(10)\end{array}$} & \multicolumn{3}{|c|}{$L_{X}$} & \multirow[b]{2}{*}{$\begin{array}{c}f_{v, 2 \mathrm{keV}} \\
\text { (14) }\end{array}$} & \multirow[b]{2}{*}{$\begin{array}{c} \pm \\
(15)\end{array}$} & \multicolumn{4}{|c|}{$\lim$} & \multirow[b]{2}{*}{$\begin{array}{l}\text { EEF } \\
(20)\end{array}$} & \multirow[b]{2}{*}{$\begin{array}{l}\text { RA } \\
(21)\end{array}$} & \multirow[b]{2}{*}{$\begin{array}{c}\text { DEC } \\
(22)\end{array}$} \\
\hline & & & & & & & & & & $\begin{array}{c}F \\
(11)\end{array}$ & $\begin{array}{c}S \\
(12)\end{array}$ & $\begin{array}{c}H \\
(13)\end{array}$ & & & $\begin{array}{l}\text { HR } \\
(16)\end{array}$ & $\begin{array}{c}F \\
(17)\end{array}$ & $\underset{(18)}{S}$ & $\begin{array}{c}H \\
(19)\end{array}$ & & & \\
\hline 1 & 1.0 & 0.0 & -0.0 & -15.0 & 0.0 & -15.7 & 0.0 & -15.1 & 0.0 & 38.6 & 37.9 & 38.5 & -33.2 & 0.0 & -2 & 5 & 1 & 5 & 0.9 & 9.7884 & 0.8779 \\
\hline $2 \mathrm{~A}$ & 0.7 & 0.0 & -0.0 & -14.6 & 0.2 & -15.4 & 0.0 & -14.7 & 0.0 & 39.0 & 38.1 & 38.9 & -32.9 & 0.2 & -2 & 0 & 5 & 5 & 0.9 & 9.7965 & 0.8677 \\
\hline 3 & 1.0 & 0.0 & -0.0 & -15.0 & 0.0 & -15.7 & 0.0 & -15.1 & 0.0 & 38.6 & 37.9 & 38.5 & -33.3 & 0.0 & -2 & 5 & 5 & 1 & 0.9 & 9.8001 & 0.8911 \\
\hline $4 \mathrm{~A}$ & 0.7 & 0.0 & -0.0 & -14.5 & 0.2 & -15.4 & 0.0 & -14.6 & 0.0 & 39.0 & 38.2 & 39.0 & -32.9 & 0.2 & -2 & 0 & 5 & 5 & 0.9 & 9.8005 & 0.8649 \\
\hline $5 \mathrm{~A}$ & 0.9 & 0.1 & -0.1 & -13.1 & 0.0 & -13.9 & 0.0 & -13.2 & 0.0 & 40.4 & 39.7 & 40.3 & -31.4 & 0.0 & 0 & 0 & 0 & 0 & 0.9 & 9.8050 & 0.8617 \\
\hline $6 A^{*}$ & 2.0 & 0.2 & -0.2 & -13.6 & 0.0 & -13.9 & 0.0 & -13.9 & 0.0 & 40.0 & 39.7 & 39.7 & -31.7 & 0.0 & 0 & 0 & 0 & 0 & 0.9 & 9.8059 & 0.8643 \\
\hline 7A & 0.7 & 0.0 & -0.0 & -14.7 & 0.2 & -15.5 & 0.0 & -14.7 & 0.0 & 38.9 & 38.1 & 38.9 & -33 & 0.2 & -2 & 0 & 5 & 5 & 9 & 9.8081 & 0.8685 \\
\hline 8 & 1.6 & 0.4 & -0.4 & -14.0 & 0.1 & -14.4 & 0.1 & -14.2 & 0.1 & 39.6 & 39.1 & 39.4 & -32.2 & 0.1 & 0 & 0 & 0 & 0 & 0.9 & 9.8082 & 0.8986 \\
\hline 9A & 2.0 & 0.4 & -0.4 & -14.0 & 0.1 & -14.3 & 0.1 & -14.3 & 0.1 & 39.6 & 39.3 & 39.3 & -32.1 & 0.1 & 0 & 0 & 0 & 0 & 0.9 & 9.8116 & 0.8800 \\
\hline 10 & 1.0 & 0.0 & -0.0 & -15.0 & 0.0 & -15.7 & 0.0 & -15.1 & 0.0 & 38.6 & 37.9 & 38.5 & -33.3 & 0.0 & -2 & 5 & 5 & 1 & 0.9 & 9.8132 & 0.8967 \\
\hline
\end{tabular}

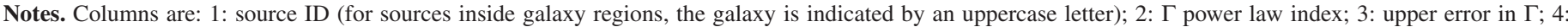

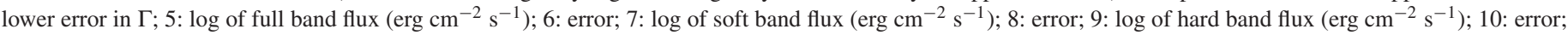

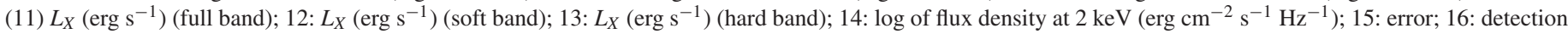

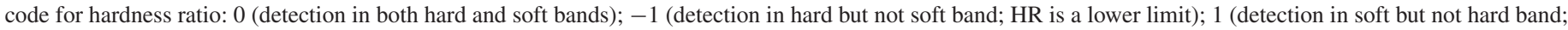

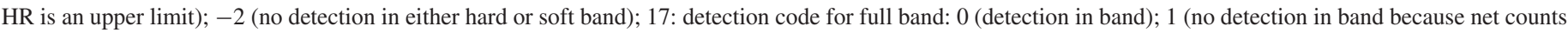

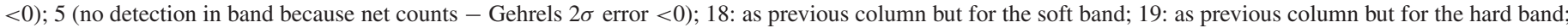
20: encircled energy fraction by AE PSF at $1.5 \mathrm{keV} ; 21$ : right ascension; 22 : declination;

(This table is available in its entirety in a machine-readable form in the online journal. A portion is shown here for guidance regarding its form and content.)

Table 3

Counts, Count Rates, and AE Detection Significance and No-source Probabilities for X-ray Point Sources in HCG 7

\begin{tabular}{|c|c|c|c|c|c|c|c|c|c|c|c|c|c|}
\hline $\begin{array}{l}\text { ID } \\
(1)\end{array}$ & $\begin{array}{c}\mathrm{c}(\mathrm{FB}) \\
(2)\end{array}$ & $\lim _{F}$ & $\begin{array}{c}\mathrm{c}(\mathrm{SB}) \\
(4)\end{array}$ & $\begin{array}{c}\lim _{S} \\
(5)\end{array}$ & $\begin{array}{c}\mathrm{c}(\mathrm{HB}) \\
(6)\end{array}$ & $\lim _{S}$ & $\begin{array}{l}\text { HR } \\
(8)\end{array}$ & $\begin{array}{c}\lim _{H R} \\
(9)\end{array}$ & $\begin{array}{c}\operatorname{cr}(\mathrm{FB}) \\
(10)\end{array}$ & $\begin{array}{c}\mathrm{cr}(\mathrm{SB}) \\
(11)\end{array}$ & $\begin{array}{c}\operatorname{cr}(\mathrm{HB}) \\
(12)\end{array}$ & $\begin{array}{c}\mathrm{AE}(\mathrm{sig}) \\
(13)\end{array}$ & $\begin{array}{c}\mathrm{AE}(P) \\
(14)\end{array}$ \\
\hline 1 & $2_{-2}^{+0}$ & 5 & $2_{-2}^{+0}$ & 1 & $2_{-2}^{+0}$ & 5 & $0.00_{-0.00}^{+0.00}$ & -2 & $0.07_{-0.07}^{+0.00}$ & $0.06_{-0.06}^{+0.00}$ & $0.07_{-0.07}^{+0.00}$ & $8 \mathrm{E}-01$ & $3 \mathrm{E}-02$ \\
\hline $2 \mathrm{~A}$ & $\begin{array}{l}5_{-2}^{+3} \\
-\end{array}$ & 0 & $2_{-2}^{+0}$ & 5 & $2_{-2}^{+0}$ & 5 & $0.00_{-0.00}^{+0.00}$ & -2 & $0.14_{-0.06}^{+0.09}$ & $0.07_{-0.07}^{+0.00}$ & $0.07_{-0.07}^{+0.00}$ & $8 \mathrm{E}-01$ & $4 \mathrm{E}-02$ \\
\hline 3 & $2_{-2}^{+0}$ & 5 & $2_{-2}^{+0}$ & 5 & $2_{-2}^{+0}$ & 1 & $0.00_{-0.00}^{+0.00}$ & -2 & $0.07_{-0.07}^{+0.00}$ & $0.07_{-0.07}^{+0.00}$ & $0.06_{-0.06}^{+0.00}$ & $-2 \mathrm{E}-01$ & $1 \mathrm{E}+00$ \\
\hline $4 \mathrm{~A}$ & $5_{-2}^{+3}$ & 0 & $2_{-2}^{+0}$ & 5 & $2_{-2}^{+0}$ & 5 & $0.00_{-0.00}^{+0.00}$ & -2 & $0.16_{-0.06}^{+0.10}$ & $0.07_{-0.07}^{+0.00}$ & $0.07_{-0.07}^{+0.00}$ & 8E-01 & $2 \mathrm{E}-02$ \\
\hline $5 \mathrm{~A}$ & $160_{-12}^{+13}$ & 0 & $88_{-9}^{+10}$ & 0 & $71_{-8}^{+9}$ & 0 & $-0.11_{-0.08}^{+0.08}$ & 0 & $4.46_{-0.35}^{+0.38}$ & $2.47_{-0.26}^{+0.29}$ & $1.99_{-0.23}^{+0.26}$ & $7 \mathrm{E}+00$ & $0 \mathrm{E}+00$ \\
\hline $6 \mathrm{~A}$ & $102_{-10}^{+11}$ & 0 & $82_{-9}^{+10}$ & 0 & $19_{-4}^{+5}$ & 0 & $-0.62_{-0.08}^{+0.09}$ & 0 & $2.85_{-0.28}^{+0.31}$ & $2.31_{-0.25}^{+0.28}$ & $0.54_{-0.12}^{+0.15}$ & $3 \mathrm{E}+00$ & $3 \mathrm{E}-22$ \\
\hline $7 \mathrm{~A}$ & $4_{-1}^{+3}$ & 0 & $2_{-2}^{+0}$ & 5 & $2_{-2}^{+0}$ & 5 & $0.00_{-0.00}^{+0.00}$ & -2 & $0.12_{-0.05}^{+0.09}$ & $0.07_{-0.07}^{+0.00}$ & $0.07_{-0.07}^{+0.00}$ & $6 \mathrm{E}-01$ & $5 \mathrm{E}-02$ \\
\hline 8 & $32_{-5}^{+6}$ & 0 & $23_{-4}^{+6}$ & 0 & $8_{-2}^{+4}$ & 0 & $-0.46_{-0.16}^{+0.19}$ & 0 & $0.91_{-0.16}^{+0.19}$ & $0.67_{-0.14}^{+0.17}$ & $0.24_{-0.08}^{+0.11}$ & $2 \mathrm{E}+00$ & $6 \mathrm{E}-12$ \\
\hline $9 \mathrm{~A}$ & $42_{-6}^{+7}$ & 0 & $34_{-5}^{+7}$ & 0 & $\begin{array}{l}7_{-2}^{+3} \\
-\end{array}$ & 0 & $-0.64_{-0.12}^{+0.15}$ & 0 & $1.18_{-0.18}^{+0.21}$ & $0.97_{-0.16}^{+0.19}$ & $0.21_{-0.08}^{+0.11}$ & $2 \mathrm{E}+00$ & $5 \mathrm{E}-09$ \\
\hline 10 & $2_{-2}^{+0}$ & 5 & $2_{-2}^{+0}$ & 5 & $2_{-2}^{+0}$ & 1 & $0.00_{-0.00}^{+0.00}$ & -2 & $0.07_{-0.07}^{+0.00}$ & $0.07_{-0.07}^{+0.00}$ & $0.06_{-0.06}^{+0.00}$ & $-9 \mathrm{E}-02$ & $1 \mathrm{E}+00$ \\
\hline
\end{tabular}

(This table is available in its entirety in a machine-readable form in the online journal. A portion is shown here for guidance regarding its form and content.)

A summary of key results from the X-ray analysis for all galaxy nuclei in this sample is presented in Table 4. In this table, the ID number in column 2 refers to the master ID running number in column 1 of Table 2. For instance, for the nucleus of HCG 7A this number is 6 (first row of Table 4). This indicates that this is the sixth source in the master X-ray catalog of group HCG 7. Since this source belongs to galaxy A and is a nuclear source, it is listed as $6 \mathrm{~A}^{\star}$ in Table 2.

Table 4 shows that there appear to be nuclear X-ray detections for 27 out of 37 HCG galaxies. According to column 6, the great majority of $\mathrm{X}$-ray sources associated with galaxy nuclei $(24 / 27$ or $89 \%)$ are soft $(H R<0)$. Compared with column 4 , we see that these are also the sources with low luminosities, $L_{X, 0.5-8.0 \mathrm{keV}}<10^{41} \mathrm{erg} \mathrm{s}^{-1}$. What about the remaining three sources? The last column indicates whether a galaxy is a good candidate for being a strong AGN host based on whether $L_{X, 0.5-8.0 \mathrm{keV}} \geqslant 10^{41} \mathrm{erg} \mathrm{s}^{-1}$. The three remaining sources (3/ 27 or $11 \%$ ) are those that fulfill this condition and are also those that have HR $>0$. These trends are also easy to see in Figure 5. The left and middle panels show histograms for

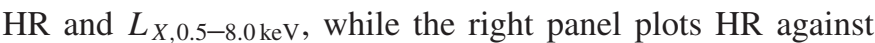
$L_{X, 0.5-8.0 \mathrm{keV}}$. Again, a small minority of positive HR high

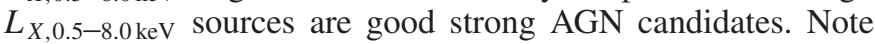
that the last column of Table 4 also appears in Table 11 (column 8), which combines multi-wavelength nuclear activity diagnostics.

\subsection{Flux Limits and Source Statistics}

Although we detect a large number of X-ray point sources in our fields, we have no a priori information about which of these sources are physically associated with HCG galaxies and are not just background AGNs. We assess the effect of AGN background contamination as follows.

The binomial probability, $P_{B}$, that a source is spurious is given by the binomial function (Broos et al. 2010)

$$
P_{B}=f_{b}\left(C_{s} ; C_{s}+C_{b},\left(1+A_{b} / A_{s}\right)^{-1}\right)
$$

where $C_{s}$ and $C_{b}$ are the number of counts observed in the source and background region in a given energy band, and 

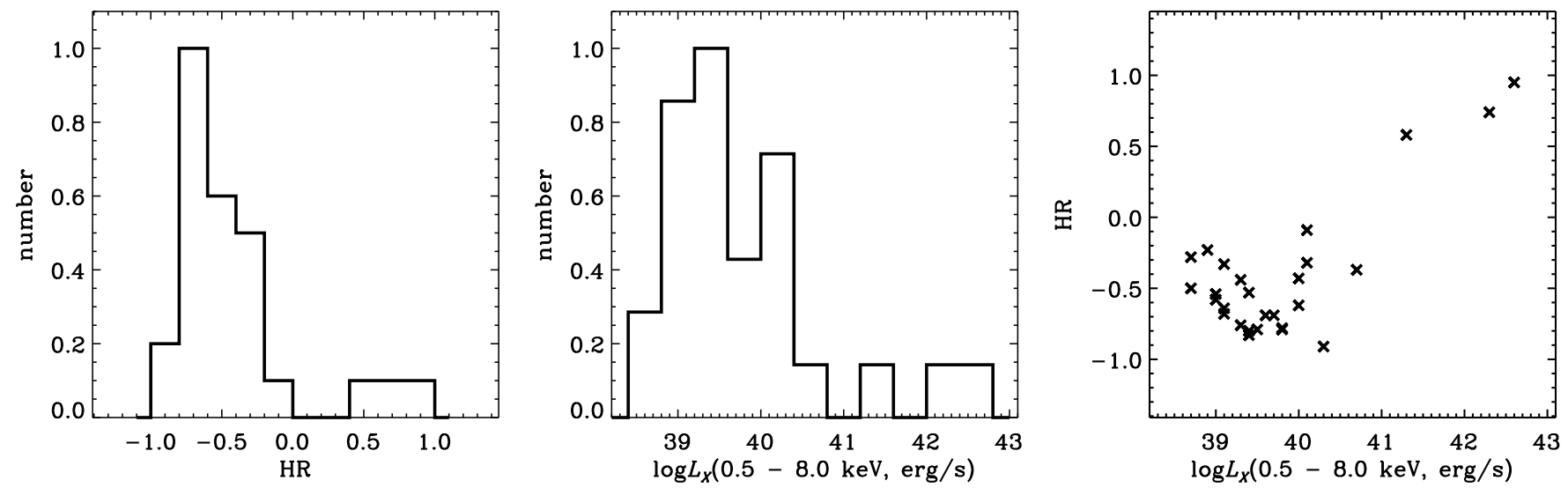

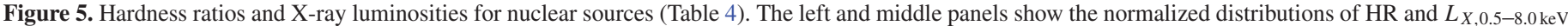

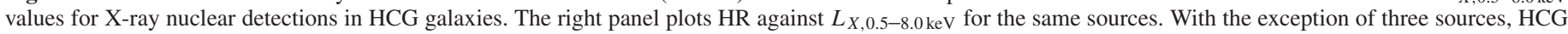
nuclei have negative HR values and low luminosities in the X-rays.

Table 4

X-Ray Analysis of HCG Nuclear Sources

\begin{tabular}{|c|c|c|c|c|c|c|}
\hline $\begin{array}{l}\text { HCG ID } \\
\text { (1) }\end{array}$ & $\begin{array}{l}\text { X-Ray ID } \\
\text { (2) }\end{array}$ & $\begin{array}{c}\Gamma \\
(3)\end{array}$ & $\begin{array}{c}L_{X, 0.5-8.0 \mathrm{keV}} \\
\text { (4) }\end{array}$ & $\begin{array}{c}\mathrm{c}(0.5-8.0 \mathrm{keV}) \\
(5)\end{array}$ & $\begin{array}{l}\text { HR } \\
\text { (6) }\end{array}$ & $\begin{array}{c}\text { Strong AGN? } \\
\text { (7) }\end{array}$ \\
\hline $7 \mathrm{~A}$ & 6 & 2.0 & 40.0 & $102_{-10}^{+11}$ & $-0.62_{-0.08}^{+0.09}$ & $\mathrm{n}$ \\
\hline 7B & 15 & 1.9 & 39.0 & $9_{-3}^{+4}$ & $-0.58_{-0.42}^{+0.00}$ & $\mathrm{n}$ \\
\hline $7 \mathrm{C}$ & 44 & 2.1 & 39.1 & $12_{-3}^{+4}$ & $-0.68_{-0.32}^{+0.00}$ & $\mathrm{n}$ \\
\hline 7D & $\ldots$ & $\ldots$ & $\ldots$ & $\ldots$ & $\ldots$ & $\ldots$ \\
\hline $16 \mathrm{~A}$ & 9 & 1.2 & 40.7 & $134_{-11}^{+12}$ & $-0.37_{-0.08}^{+0.09}$ & $\mathrm{n}$ \\
\hline $16 \mathrm{~B}$ & 4 & -0.7 & 41.3 & $189_{-13}^{+14}$ & $0.58_{-0.07}^{+0.06}$ & y \\
\hline $16 \mathrm{C}$ & 44 & 2.0 & 39.7 & $24_{-4}^{+6}$ & $-0.69_{-0.15}^{+0.21}$ & $\mathrm{n}$ \\
\hline $16 \mathrm{D}$ & 57 & 2.4 & 39.8 & $37_{-6}^{+7}$ & $-0.79_{-0.10}^{+0.15}$ & $\mathrm{n}$ \\
\hline $22 \mathrm{~A}$ & 77,76 & $1.7(1.1)$ & $38.7(38.7)$ & $9_{-3}^{+4}\left(7_{-2}^{+}\right)$ & $-0.50_{-0.50}^{+0.00}\left(-0.23_{-0.77}^{+0.00}\right)$ & $\mathrm{n}$ \\
\hline $22 \mathrm{~B}$ & 22 & 1.2 & 38.7 & $7_{-2}^{+3}$ & $-0.28_{-0.72}^{+0.00}$ & $\mathrm{n}$ \\
\hline $22 \mathrm{C}$ & $\ldots$ & $\ldots$ & $\ldots$ & $\ldots$ & $\ldots$ & $\mathrm{n}$ \\
\hline $31 \mathrm{ACE}$ & 40,38 & $1.3(1.1)$ & $40.1(40.3)$ & $73_{-8}^{+9}\left(128_{-11}^{+1}\right)$ & $-0.32_{-0.12}^{+0.12}\left(-0.21_{-0.09}^{+0.09}\right)$ & $\mathrm{n}$ \\
\hline 31B & $\ldots$ & $\ldots$ & $\ldots$ & $\ldots$ & $\ldots$ & $\ldots$ \\
\hline $31 \mathrm{~F}$ & $\ldots$ & $\ldots$ & $\ldots$ & $\ldots$ & $\ldots$ & $\ldots$ \\
\hline $31 \mathrm{G}$ & 63 & 1.6 & 40.0 & $72_{-8}^{+9}$ & $-0.43_{-0.11}^{+0.12}$ & $\mathrm{n}$ \\
\hline $31 \mathrm{Q}$ & 41 & 1.1 & 38.9 & $4_{-2}^{+3}$ & $-0.23_{-0.77}^{+0.00}$ & $\mathrm{n}$ \\
\hline $42 \mathrm{~A}$ & 18 & 3.3 & 40.3 & $178_{-13}^{+14}$ & $-0.91_{-0.03}^{+0.04}$ & $\mathrm{n}$ \\
\hline $42 \mathrm{~B}$ & $\ldots$ & $\ldots$ & $\ldots$ & $\ldots$ & $\ldots$ & $\ldots$ \\
\hline $42 \mathrm{C}$ & 6 & 2.4 & 39.3 & $19_{-4}^{+5}$ & $-0.76_{-0.24}^{+0.00}$ & $\mathrm{n}$ \\
\hline 42D & .. & $\ldots$ & $\ldots$ & $\ldots$ & $\ldots$ & $\ldots$ \\
\hline $59 \mathrm{~A}$ & 50 & 0.8 & 40.1 & $43_{-6}^{+7}$ & $-0.09_{-0.16}^{+0.17}$ & $\mathrm{n}$ \\
\hline $59 \mathrm{~B}$ & 26 & 1.5 & 39.3 & $10_{-3}^{+4}$ & $-0.44_{-0.56}^{+0.00}$ & $\mathrm{n}$ \\
\hline $59 \mathrm{C}$ & $\ldots$ & $\ldots$ & $\ldots$ & $\ldots$ & $\ldots$ & $\ldots$ \\
\hline 59D & $\ldots$ & $\ldots$ & $\ldots$ & $\ldots$ & $\ldots$ & $\ldots$ \\
\hline $62 \mathrm{~A}$ & 83 & 2.5 & 39.5 & $133_{-11}^{+12}$ & $-0.79_{-0.05}^{+0.07}$ & $\mathrm{n}$ \\
\hline $62 \mathrm{~B}$ & 67 & 2.6 & 39.4 & $116_{-10}^{+11}$ & $-0.80_{-0.06}^{+0.07}$ & $\mathrm{n}$ \\
\hline $62 \mathrm{C}$ & 115 & 1.7 & 39.0 & $33_{-5}^{+6}$ & $-0.54_{-0.15}^{+0.18}$ & $\mathrm{n}$ \\
\hline $62 \mathrm{D}$ & $\ldots$ & $\ldots$ & $\ldots$ & $\ldots$ & $\ldots$ & $\ldots$ \\
\hline $90 \mathrm{~A}$ & 88 & -1.1 & 42.6 & $23361_{-152}^{+153}$ & $0.95_{-0.00}^{+0.00}$ & $\mathrm{y}$ \\
\hline $90 \mathrm{~B}$ & 164 & 2.3 & 39.1 & $31_{-5}^{+6}$ & $-0.64_{-0.14}^{+0.18}$ & $\mathrm{n}$ \\
\hline $90 \mathrm{C}$ & 108 & 1.5 & 39.1 & $23_{-4}^{+5}$ & $-0.33_{-0.21}^{+0.23}$ & $\mathrm{n}$ \\
\hline 90D & $\ldots$ & $\ldots$ & $\ldots$ & $\ldots$ & $\ldots$ & $\ldots$ \\
\hline $92 \mathrm{~B}$ & 46 & 1.8 & 39.4 & $19_{-4}^{+5}$ & $-0.53_{-0.21}^{+0.25}$ & $\mathrm{n}$ \\
\hline $92 \mathrm{C}$ & 94 & -1.1 & 42.3 & $3375_{-58}^{+59}$ & $0.74_{-0.01}^{+0.01}$ & $\mathrm{y}$ \\
\hline $92 \mathrm{D}$ & 36 & 2.6 & 39.8 & $75_{-8}^{+9}$ & $-0.78_{-0.07}^{+0.09}$ & $\mathrm{n}$ \\
\hline $92 \mathrm{E}$ & 22 & 2.3 & 39.6 & $40_{-6}^{+7}$ & $-0.69_{-0.12}^{+0.15}$ & $\mathrm{n}$ \\
\hline $92 \mathrm{~F}$ & 145 & 2.9 & 39.4 & $30_{-5}^{+6}$ & $-0.83_{-0.17}^{+0.00}$ & $\mathrm{n}$ \\
\hline
\end{tabular}

Notes. Columns are: 1: HCG galaxy ID; 2: X-ray source ID in master list; $3: \Gamma$ value from the hardness ratio; 4: $\log L_{X}$ in the full band; 5: net counts in the full band; 6: hardness ratio; 7: X-rays indicating strong AGNs $\left(L_{X, 0.5-8.0 \mathrm{keV}} \geqslant 10^{41} \mathrm{erg} \mathrm{s}^{-1}\right)$. 
Table 5

Flux Limit Estimates and X-Ray Point Sources Associated with Galaxies

\begin{tabular}{|c|c|c|c|c|c|c|c|c|c|c|}
\hline (1) & 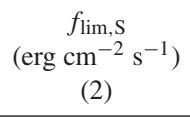 & $\begin{array}{c}L_{X, \lim , S} \\
\left(\mathrm{erg} \mathrm{s}^{-1}\right) \\
(3)\end{array}$ & $\begin{array}{c}N_{\text {obs, }} \\
\text { (4) }\end{array}$ & $\begin{array}{c}N_{\text {exc }, \mathrm{S}} \\
(5)\end{array}$ & $\begin{array}{l}P_{S} \\
(6)\end{array}$ & 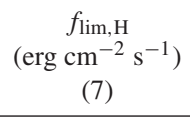 & $\begin{array}{c}L_{X, \lim , \mathrm{H}} \\
\left(\mathrm{erg} \mathrm{s}^{-1}\right) \\
(8)\end{array}$ & $\begin{array}{c}N_{\text {obs, } \mathrm{H}} \\
\text { (9) }\end{array}$ & $\begin{array}{c}N_{\text {exc }, \mathrm{H}} \\
(10)\end{array}$ & (11) \\
\hline HCG 7 A & $5.8 \times 10^{16}$ & $2.2 \times 10^{38}$ & 4 & 3.5 & 0.9 & $2.0 \times 10^{15}$ & $7.6 \times 10^{38}$ & 3 & 2.3 & 0.8 \\
\hline HCG 7 B & $2.9 \times 10^{16}$ & $1.1 \times 10^{38}$ & 3 & 2.5 & 0.8 & $1.5 \times 10^{15}$ & $5.7 \times 10^{38}$ & 0 & -0.5 & 0.0 \\
\hline $\mathrm{HCG} 7 \mathrm{C}$ & $3.9 \times 10^{16}$ & $1.5 \times 10^{38}$ & 6 & 5.5 & 0.9 & $2.5 \times 10^{15}$ & $9.5 \times 10^{38}$ & 2 & 1.6 & 0.8 \\
\hline HCG 16 A & $1.9 \times 10^{15}$ & $6.6 \times 10^{38}$ & 2 & 1.9 & 0.9 & $5.8 \times 10^{15}$ & $2.0 \times 10^{39}$ & 2 & 1.9 & 0.9 \\
\hline HCG $16 \mathrm{C}$ & $3.9 \times 10^{15}$ & $1.3 \times 10^{39}$ & 6 & 5.9 & 1.0 & $5.8 \times 10^{15}$ & $2.0 \times 10^{39}$ & 2 & 1.8 & 0.9 \\
\hline HCG $16 \mathrm{D}$ & $1.4 \times 10^{15}$ & $4.7 \times 10^{38}$ & 1 & 0.9 & 0.9 & $4.4 \times 10^{15}$ & $1.5 \times 10^{39}$ & 1 & 0.8 & 0.8 \\
\hline HCG $22 \mathrm{~A}$ & $4.4 \times 10^{16}$ & $6.9 \times 10^{37}$ & 15 & 13.9 & 0.9 & $1.7 \times 10^{15}$ & $2.7 \times 10^{38}$ & 4 & 2.7 & 0.7 \\
\hline HCG 22 B & $3.3 \times 10^{16}$ & $5.2 \times 10^{37}$ & 1 & 0.8 & 0.8 & $1.7 \times 10^{15}$ & $2.7 \times 10^{38}$ & 0 & -0.2 & 0.0 \\
\hline HCG 31 ACE & $1.1 \times 10^{15}$ & $4.3 \times 10^{38}$ & 9 & 8.9 & 1.0 & $2.6 \times 10^{15}$ & $1.0 \times 10^{39}$ & 9 & 8.8 & 1.0 \\
\hline HCG $31 \mathrm{G}$ & $5.8 \times 10^{16}$ & $2.3 \times 10^{38}$ & 5 & 4.9 & 1.0 & $2.1 \times 10^{15}$ & $8.3 \times 10^{38}$ & 3 & 2.9 & 1.0 \\
\hline HCG $31 \mathrm{Q}$ & $2.9 \times 10^{16}$ & $1.2 \times 10^{38}$ & 1 & 1.0 & 1.0 & $1.5 \times 10^{15}$ & $6.2 \times 10^{38}$ & 0 & 0.0 & 0.0 \\
\hline $\mathrm{HCG} 42 \mathrm{C}$ & $1.2 \times 10^{15}$ & $5.6 \times 10^{38}$ & 4 & 3.7 & 0.9 & $3.4 \times 10^{15}$ & $1.6 \times 10^{39}$ & 1 & 0.7 & 0.7 \\
\hline HCG 59 A & $5.4 \times 10^{16}$ & $2.6 \times 10^{38}$ & 1 & 0.9 & 0.9 & $1.4 \times 10^{15}$ & $6.9 \times 10^{38}$ & 1 & 0.8 & 0.8 \\
\hline HCG 59 B & $2.7 \times 10^{16}$ & $1.3 \times 10^{38}$ & 3 & 2.9 & 1.0 & $1.4 \times 10^{15}$ & $6.9 \times 10^{38}$ & 0 & -0.1 & 0.0 \\
\hline HCG 62 A & $3.5 \times 10^{15}$ & $1.7 \times 10^{39}$ & 11 & 10.9 & 1.0 & $1.2 \times 10^{15}$ & $5.9 \times 10^{38}$ & 6 & 5.6 & 0.9 \\
\hline HCG 62 B & $1.2 \times 10^{15}$ & $5.9 \times 10^{38}$ & 6 & 5.9 & 1.0 & $8.7 \times 10^{16}$ & $4.3 \times 10^{38}$ & 4 & 3.7 & 0.9 \\
\hline HCG 62 C & $2.5 \times 10^{16}$ & $1.2 \times 10^{38}$ & 9 & 8.7 & 1.0 & $6.5 \times 10^{16}$ & $3.2 \times 10^{38}$ & 8 & 7.5 & 0.9 \\
\hline HCG $90 \mathrm{~A}$ & $1.0 \times 10^{15}$ & $1.4 \times 10^{38}$ & 1 & 0.7 & 0.7 & $2.2 \times 10^{14}$ & $3.1 \times 10^{39}$ & 1 & 1.0 & 1.0 \\
\hline HCG 90 B & $6.7 \times 10^{16}$ & $9.3 \times 10^{37}$ & 7 & 6.8 & 1.0 & $1.7 \times 10^{15}$ & $2.3 \times 10^{38}$ & 2 & 1.6 & 0.8 \\
\hline HCG $90 \mathrm{C}$ & $4.5 \times 10^{16}$ & $6.2 \times 10^{37}$ & 5 & 4.8 & 1.0 & $1.3 \times 10^{15}$ & $1.8 \times 10^{38}$ & 5 & 4.8 & 1.0 \\
\hline HCG 92 B & $1.8 \times 10^{16}$ & $1.7 \times 10^{38}$ & 2 & 1.9 & 0.9 & $6.5 \times 10^{16}$ & $6.1 \times 10^{38}$ & 1 & 0.9 & 0.9 \\
\hline HCG $92 \mathrm{C}$ & $1.3 \times 10^{15}$ & $1.3 \times 10^{39}$ & 2 & 2.0 & 1.0 & $3.1 \times 10^{15}$ & $2.9 \times 10^{39}$ & 2 & 2.0 & 1.0 \\
\hline HCG 92 D & $2.1 \times 10^{16}$ & $2.0 \times 10^{38}$ & 2 & 1.9 & 0.9 & $8.1 \times 10^{16}$ & $7.6 \times 10^{38}$ & 1 & 0.9 & 0.9 \\
\hline HCG 92 E & $1.8 \times 10^{16}$ & $1.7 \times 10^{38}$ & 2 & 1.8 & 0.9 & $8.1 \times 10^{16}$ & $7.6 \times 10^{38}$ & 1 & 0.8 & 0.8 \\
\hline HCG $92 \mathrm{~F}$ & $3.7 \times 10^{16}$ & $3.5 \times 10^{38}$ & 1 & 0.8 & 0.8 & $2.9 \times 10^{15}$ & $2.7 \times 10^{39}$ & 0 & -0.1 & 0.0 \\
\hline
\end{tabular}

Notes. Columns are: 1: HCG galaxy ID; 2: flux limit estimate in the soft band; 3: luminosity limit estimate in the soft band; 4: number of detected point sources inside the galaxy region; 5 : number of point sources in the soft band and inside the galaxy region that are in excess of the number expected from the background $\log N-\log S ; 6$ : probability estimate that in the soft band point sources detected in this galaxy belong to the galaxy and are not background AGNs (Column 5/Column 4); 7: same as Column 2 but for the hard band; 8 same as Column 3 but for the hard band; 9 same as Column 4 but for the hard band; 10: same as Column 5 but for the hard band; 11: same as Column 6 but for the hard band.

$A_{s}, A_{b}$ are the areas of the source and background regions. In other words, sources with values of $P_{B}$ less than a given threshold value may be considered detections. We adopt the threshold $P_{B}=0.004$ established by Xue et al. (2011) and use local background information for each detected nuclear source to establish a detectability limit in terms of counts, fluxes, and luminosities in the soft and hard bands at the location of each galaxy on the ACIS CCD. Each of our nuclear sources has associated background and source extraction regions with measured background counts. The advantage of using these regions, together with measured background counts, is that they have been constructed and measured by ACIS EXTRACT by taking into account the size of the Chandra PSF at the location of the particular source on the CCD. Thus the detection limits that we calculate are local, position-dependent, and specific to each galaxy. We fix $C_{b}$ to the locally measured background counts and start by setting the source counts $C_{s}=0$, in which case $P_{B} \gg 0.004$ (a source with no source counts must be spurious). We then iteratively increase $C_{s}$ to estimate the minimum number of source counts, $C_{s, \mathrm{lim}}$, required to reach our chosen probability threshold of 0.004 . We convert the estimated $C_{s, \text { lim }}$ value to a flux limit by assuming a power law spectrum with $\Gamma=1.4$ (Hickox \& Markevitch 2006; Steffen et al. 2007) and the Galactic $N_{H}$ value for each galaxy group in PIMMs (Mukai 1993). The flux and corresponding luminosity limits for the soft and hard bands are shown in columns 2 and 3 (soft band) and 7 and 8 (hard band) of Table 5. As expected, the estimated limit fluxes are lower in the soft band due to the higher sensitivity of ACIS in this regime.

We use these flux limits to estimate how many background AGNs we expect to see inside the galaxy regions of our galaxies. We use the " $\log N-\log S$ " relation of Cappelluti et al. (2007) which relates the number of detected point sources per angular area in the soft and hard bands as a function of flux, established over $2 \mathrm{deg}^{2}$ in the COSMOS field. We thus estimate for each band the expected total number of background AGNs that would be detected over the area of each of our galaxy regions. These regions are determined following Tzanavaris et al. (2010) who use mid-infrared defined galaxy regions (Johnson et al. 2007) for their global galaxy photometry. For each band and galaxy, we then compare the number of expected AGNs to the number of detected sources, $N_{\mathrm{obs}, \mathrm{S}}$ and $N_{\mathrm{obs}, \mathrm{H}}$ (columns 4 and 9 in Table 5), and calculate the number of detected sources which are in excess of the expected background number, $N_{\mathrm{exc}, \mathrm{S}}$ and $N_{\text {exc,H }}$ (columns 5 and 10 in Table 5). The ratio of the number of excess sources over the total number of observed sources is a rough estimate of the probability that a source detected inside a galaxy region is not a background AGN. Although this method cannot tell us whether a specific source is likely to be a background source or not, it does provide an overall estimate of 
Table 6

Swift UVOT Magnitude Limits for HCG Fields

\begin{tabular}{lccc}
\hline \hline HCG ID & $\begin{array}{c}u v w 2 \\
(2030 \AA)\end{array}$ & $\begin{array}{c}u v m 2 \\
(2231 \AA)\end{array}$ & $\begin{array}{c}u v w 1 \\
(2634 \AA)\end{array}$ \\
$(1)$ & $(2)$ & $(3)$ & $(4)$ \\
\hline 7 & 20.5 & 20.4 & 20.6 \\
16 & 21.6 & 20.6 & 20.6 \\
22 & 20.2 & 20.7 & 20.4 \\
31 & 20.4 & 20.3 & 20.5 \\
42 & 20.5 & 20.1 & 20.9 \\
59 & 20.7 & 20.2 & 21.1 \\
62 & 20.6 & 20.3 & 20.9 \\
90 & 22.6 & 22.8 & 21.6 \\
92 & 20.9 & 21.0 & 20.3 \\
\hline
\end{tabular}

Notes. Columns are: 1: HCG field ID; $2-4$ : magnitude limit estimates for the $u v w 2$ (effective wavelength $2030 \AA$ ), $u v m 2$ (2231 $\AA$ ), $u v w 1$ (2634 A) UVOT filters.

whether background contamination may be a serious concern for our galaxies. The probability estimates in Table 5 (columns 6 and 11) are generally quite high. Note that in these columns values equal to zero are simply due to non-detections in one band. Otherwise, rounded values range from 0.7 to 1.0 , giving us confidence that our point source detections are likely due to HCG galaxies.

\section{UV DATA AND DATA ANALYSIS}

\subsection{Data}

For all galaxies in this sample we use three-band UV data obtained with UVOT on NASA's Swift Gamma-ray Burst Explorer (Gehrels et al. 2004). Details on the telescope, filters, observations, and data reduction can be found in Tzanavaris et al. (2010). Magnitude limits for each HCG field are estimated using the zero points specific to each UVOT filter (Poole et al. 2008) and shown in Table 6. For quick reference, we mention here that the three UV bands used are $u v w 2$, $u v m 2$, and $u v w 1$, with effective wavelengths 2030, 2231, and $2634 \AA$, respectively.

As mentioned, in the present work we do include UVOT data for HCG 90. These were excluded in Tzanavaris et al. (2010) as some of the HCG galaxies were not fully covered by the stacked UVOT exposures. A careful re-analysis of the UVOT data for this group reveals that they consist of two exposure "stacks". The HCG galaxies are not covered by 3 out of 11 individual exposures in stack 00053602001 . We thus combined the eight useful exposures of this stack with the second stack. The corrected total exposure times for HCG 90 and the $u v w 2, u v m 2$, and $u v w 1$ filters are now 7387s, 6732s, and 5525s (c.f. 8601s, 7946s, 6663s in Tzanavaris et al. (2010, Table 3)).

We also include UVOT data for HCG 92 (Stephan's Quintet). The UVOT observation IDs for this group are 00035083005 , 00035083007, 00035083008, and 00035083009, with total exposure times for the $u v w 2, u v m 2$, and $u v w 1$ filters of $3054 \mathrm{~s}$, 3214 s, and 1007 s, respectively.

\subsection{UV Nuclear Photometry}

Tzanavaris et al. (2010) carried out galaxy-wide photometry for their HCG galaxies in order to calculate galaxy star formation rates. In this paper we are interested in comparing nuclear fluxes in the UV and X-ray regime and thus perform nuclear photometry as described below.
Using the $u v w l(\sim 2600 \AA)$ image, we define circular source regions of radius $5^{\prime \prime}$, centered at the central intensity peak of each galaxy. The choice of radius is dictated both by the UVOT PSF (2".37, FWHM, for $u v w 1$, Breeveld et al. 2010) and the fact that the UVOT count rate to flux conversion factors have been calibrated for such a radius. We use the $u v w l$ image because its effective wavelength is closest to the one traditionally used to estimate the X-ray-to-UV spectral index

$$
\alpha_{\mathrm{OX}} \equiv 0.380 \log \left(L_{v, 2 \mathrm{keV}} / L_{v, u v w 1}\right)
$$

sometimes referred to as "X-ray loudness" (Tananbaum et al. 1979), or the X-ray-to-"optical" spectral index. ${ }^{13}$ Note that, since the effective wavelength of the $u v w 1$ filter is $\sim 100 \AA$ redward of $2500 \AA$, this is very close but not identical to frequent definitions of this index which use the $2500 \AA$ luminosity instead. However, given that the spectral slope is essentially flat in the near-to-far UV spectral region (Kennicutt 1998), we expect this discrepancy to have a negligible effect, especially given the scatter in our $\alpha_{\text {OX }}$ results (more than an order of magnitude, Section 5.3).

At this point we should mention a possible adverse consequence of the UV resolution and photometric aperture. Since the resolution is worse and the aperture is larger than the corresponding quantities for the Chandra data, and given that we are interested in detecting a possible signature of AGN activity, there is a risk of an AGN signal being diluted if it is weak and there is significant star formation. This issue will be less important for earlier type galaxies where star formation is unlikely to play a major role. We will come back to this effect later in the paper, but at this point we are cautioning that it is difficult to quantify properly.

To estimate background emission we construct background regions interactively to ensure that no emission from either galaxies or foreground stars was included. In a minority of cases, concentric annuli at radii $50^{\prime \prime}$ and $60^{\prime \prime}$ from the source centers are appropriate. However, compact group galaxies are often very close to each other so that in most cases we have to manually construct a background region to avoid contamination. We then obtain net count rates in all three UV bands. Finally, using the UVOT-specific flux conversion factors (Poole et al. 2008), we obtain flux and luminosity densities for all galaxies (see Table 7). The tabulated values have been corrected for Galactic extinction using the maps of Schlegel et al. (1998) and the extinction curve of Cardelli et al. (1989).

For each galaxy, UV fluxes and luminosities have further been corrected for intrinsic extinction by using the UV and $24 \mu \mathrm{m}$ SFR components in Tzanavaris et al. (2010) and assuming

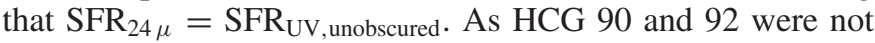
analyzed in that work, for these groups we correct for extinction by adopting the highest $A_{\mathrm{UV}}$ value for $\mathrm{HCG} 92$ in $\mathrm{Xu}$ et al. (2005), i.e., $A_{\mathrm{UV}}=2$.

\section{MULTI-WAVELENGTH NUCLEAR ANALYSIS}

We investigate the nature of nuclear activity in our HCG galaxies by combining diagnostics using the X-ray, UV, optical, and radio regions. An overview of the main multi-wavelength results is presented in Table 11, which we discuss in greater detail later.

\footnotetext{
${ }^{13}$ Note that with this definition, in this paper lower $\alpha_{\mathrm{OX}}$ values are more
} negative. 
Table 7

Swift UVOT Nuclear Photometry

\begin{tabular}{|c|c|c|c|c|}
\hline $\begin{array}{l}\text { HCG ID } \\
\text { (1) }\end{array}$ & $\begin{array}{c}f_{v}(2030 \AA) \\
(2)\end{array}$ & $\begin{array}{c}f_{v}(2231 \AA) \\
(3)\end{array}$ & $\begin{array}{c}f_{v}(2634 \AA) \\
(4)\end{array}$ & $\begin{array}{c}v L_{v}(2634 \AA) \\
(5)\end{array}$ \\
\hline $7 \mathrm{~A}$ & $0.13 \pm 0.01$ & $0.16 \pm 0.01$ & $0.27 \pm 0.01$ & 42.1 \\
\hline $7 B$ & $0.06 \pm 0.00$ & $0.05 \pm 0.01$ & $0.17 \pm 0.01$ & 41.9 \\
\hline $7 \mathrm{C}$ & $0.20 \pm 0.01$ & $0.24 \pm 0.01$ & $0.26 \pm 0.01$ & 42.1 \\
\hline 7D & $0.14 \pm 0.01$ & $0.16 \pm 0.01$ & $0.17 \pm 0.01$ & 41.9 \\
\hline $16 \mathrm{~A}$ & $0.58 \pm 0.03$ & $0.71 \pm 0.03$ & $0.97 \pm 0.04$ & 42.6 \\
\hline $16 \mathrm{~B}$ & $0.04 \pm 0.00$ & $0.03 \pm 0.01$ & $0.12 \pm 0.01$ & 41.7 \\
\hline $16 \mathrm{C}$ & $1.32 \pm 0.05$ & $1.52 \pm 0.05$ & $1.77 \pm 0.07$ & 42.8 \\
\hline $16 \mathrm{D}$ & $0.10 \pm 0.01$ & $0.11 \pm 0.01$ & $0.20 \pm 0.01$ & 41.9 \\
\hline $22 \mathrm{~A}$ & $0.12 \pm 0.01$ & $0.10 \pm 0.01$ & $0.29 \pm 0.02$ & 41.7 \\
\hline $22 B$ & $0.04 \pm 0.00$ & $0.04 \pm 0.01$ & $0.07 \pm 0.01$ & 41.1 \\
\hline $22 \mathrm{C}$ & $0.11 \pm 0.01$ & $0.12 \pm 0.01$ & $0.10 \pm 0.01$ & 41.3 \\
\hline $31 \mathrm{ACE}$ & $2.74 \pm 0.13$ & $3.15 \pm 0.11$ & $2.59 \pm 0.11$ & 43.1 \\
\hline $31 \mathrm{~B}$ & $0.35 \pm 0.02$ & $0.42 \pm 0.02$ & $0.37 \pm 0.02$ & 42.2 \\
\hline $31 \mathrm{~F}$ & $0.17 \pm 0.02$ & $0.19 \pm 0.01$ & $0.17 \pm 0.01$ & 41.9 \\
\hline $31 \mathrm{G}$ & $1.33 \pm 0.07$ & $1.50 \pm 0.06$ & $1.28 \pm 0.06$ & 42.8 \\
\hline $31 Q$ & $0.14 \pm 0.01$ & $0.15 \pm 0.01$ & $0.15 \pm 0.01$ & 41.8 \\
\hline $42 \mathrm{~A}$ & $0.18 \pm 0.01$ & $0.17 \pm 0.01$ & $0.36 \pm 0.02$ & 42.3 \\
\hline $42 B$ & $0.05 \pm 0.01$ & $0.03 \pm 0.01$ & $0.10 \pm 0.01$ & 41.7 \\
\hline $42 \mathrm{C}$ & $0.05 \pm 0.01$ & $0.04 \pm 0.01$ & $0.14 \pm 0.01$ & 41.9 \\
\hline $42 \mathrm{D}$ & $0.02 \pm 0.00$ & $0.02 \pm 0.01$ & $0.05 \pm 0.01$ & 41.4 \\
\hline $59 \mathrm{~A}$ & $0.06 \pm 0.01$ & $0.07 \pm 0.01$ & $0.14 \pm 0.01$ & 41.9 \\
\hline 59B & $0.02 \pm 0.00$ & $0.02 \pm 0.01$ & $0.05 \pm 0.01$ & 41.5 \\
\hline $59 \mathrm{C}$ & $0.08 \pm 0.01$ & $0.09 \pm 0.01$ & $0.09 \pm 0.01$ & 41.7 \\
\hline 59D & $0.19 \pm 0.01$ & $0.21 \pm 0.01$ & $0.17 \pm 0.01$ & 42.0 \\
\hline $62 \mathrm{~A}$ & $0.09 \pm 0.01$ & $0.08 \pm 0.01$ & $0.20 \pm 0.01$ & 42.1 \\
\hline $62 B$ & $0.07 \pm 0.01$ & $0.06 \pm 0.01$ & $0.17 \pm 0.01$ & 42.0 \\
\hline $62 \mathrm{C}$ & $0.03 \pm 0.00$ & $0.03 \pm 0.01$ & $0.09 \pm 0.01$ & 41.7 \\
\hline $62 \mathrm{D}$ & $0.02 \pm 0.00$ & $0.02 \pm 0.01$ & $0.06 \pm 0.01$ & 41.5 \\
\hline $90 \mathrm{~A}$ & $0.05 \pm 0.00$ & $0.04 \pm 0.01$ & $0.11 \pm 0.01$ & 41.3 \\
\hline $90 \mathrm{~B}$ & $0.15 \pm 0.01$ & $0.12 \pm 0.01$ & $0.36 \pm 0.02$ & 41.8 \\
\hline $90 \mathrm{C}$ & $0.12 \pm 0.01$ & $0.10 \pm 0.01$ & $0.32 \pm 0.02$ & 41.7 \\
\hline $90 \mathrm{D}$ & $0.08 \pm 0.01$ & $0.08 \pm 0.01$ & $0.14 \pm 0.01$ & 41.3 \\
\hline $92 \mathrm{~B}$ & $0.04 \pm 0.01$ & $0.05 \pm 0.01$ & $0.32 \pm 0.02$ & 42.5 \\
\hline $92 \mathrm{C}$ & $0.03 \pm 0.01$ & $0.04 \pm 0.01$ & $0.17 \pm 0.01$ & 42.3 \\
\hline $92 \mathrm{D}$ & $0.07 \pm 0.01$ & $0.06 \pm 0.01$ & $0.38 \pm 0.02$ & 42.6 \\
\hline $92 \mathrm{E}$ & $0.04 \pm 0.01$ & $0.04 \pm 0.01$ & $0.29 \pm 0.02$ & 42.5 \\
\hline $92 \mathrm{~F}$ & $0.02 \pm 0.01$ & $0.02 \pm 0.01$ & $0.10 \pm 0.01$ & 42.0 \\
\hline
\end{tabular}

Notes. Columns are: 1: HCG nucleus ID; 2-4): flux densities (mJy) for the $u v w 2$ (effective wavelength $2030 \AA$ ), $u v m 2$ (2231 $)$ ), and $u v w 1$ (2634 $)$ UVOT filters (corrected for Galactic extinction only); 5: $\log$ luminosity $\left(\mathrm{erg} \mathrm{s}^{-1}\right)$ for $u v w 1$ filter.

\subsection{Optical}

\subsubsection{Emission Line Ratio Classifications}

M10 have carried out spectroscopic observations and obtained emission line ratios for $200 \mathrm{HCG}$ galaxies. They also obtained emission line ratios from archival spectra and the literature for another $70 \mathrm{HCG}$ galaxies, bringing the total to 270. Their primary classification criterion is the location of a galaxy in the $[\mathrm{O}$ III $] / \mathrm{H} \beta$ versus $[\mathrm{N}$ II] $/ \mathrm{H} \alpha$ diagram of $\mathrm{K} 06$ (hereafter K06-a; see K06 Figures 1(a) and 4(a) and also Baldwin et al. 1981; Veilleux \& Osterbrock 1987). As explained by K06, galaxies located below the Kauffmann et al. (2003, hereafter $\mathrm{Ka} 03$ ) line (lower curve) are purely star-forming, while galaxies above the Kewley et al. (2001, hereafter Ke01) line are purely AGNs. Galaxies that fall between the two lines are considered composite or transition objects (Ho et al. 1997) in which circumnuclear star formation effectively dilutes the high-ionization emission line ratio signal. Note that this diagram cannot distinguish between LINERs and AGNs. M10 assume that LINERs are just an AGN subcategory and use this diagram to separate SF from AGN systems. Although K06 also consider LINERs to be AGNs, their additional $[\mathrm{O} \mathrm{III}] / \mathrm{H} \beta$ versus $[\mathrm{S} \mathrm{II}] / \mathrm{H} \alpha$ and $[\mathrm{O} \mathrm{III}] / \mathrm{H} \beta$ versus $[\mathrm{O} \mathrm{I}] / \mathrm{H} \alpha$ diagrams can be used to establish a well defined dividing line (their Figures 4(b) and (c), hereafter K06-b and K06-c, respectively) separating galaxies dominated by LINERs from those dominated by Seyfert (i.e., AGN) activity. The distinction between LINERs and AGNs is not a mere matter of semantics for two reasons. First, although a majority of LINERs harbor weak AGNs, not all LINERs have AGNs. Second, the energetics of LINERs cannot be understood in terms of AGN activity, as any weak AGNs in LINERs cannot fully account for the observed emission lines. Thus LINERs, even those that host weak AGNs, are not scaled-down AGNs; in particular, they are not just LLAGNs. They should be considered an activity class in their own right in addition to star-forming and AGN systems.

We thus use all three diagnostic diagrams in K06 and the emission line ratios of M10 as our primary criteria for obtaining new nuclear classifications for galaxies in our sample. Our classification scheme explicitly includes LINERs. Our new classifications are presented in Table 8 and Figure 6. Columns 2, 3 , and 4 of this table give the classification based on each of the emission line ratio diagrams K06-a, -b, and -c. As we do not consider LINERs to be just an AGN subclass, we can only use K06-a to classify galaxies as SF, non-SF (either Seyfert or LINER), or TO. We use K06-b and K06-c to classify galaxies as $\mathrm{SF}, \mathrm{AGN}$, or LINER. Our final classification is given in column 5 and is based on the results of the previous three columns. If in any of the diagrams a galaxy falls on the dividing line, this is a borderline case indicated by a question mark in Table 8 .

Finally, for three galaxies in our sample there is only $[\mathrm{NII}] / \mathrm{H} \alpha$ information. In this case we adopt the classification criterion of M10, who classify galaxies with $\log ([\mathrm{NII}] / \mathrm{H} \alpha) \leqslant-0.4$ as $\mathrm{SF}$, those with $\log ([\mathrm{N} \mathrm{II}] / \mathrm{H} \alpha)>$ -0.1 as AGN, and those in between as TO (Stasińska et al. 2006). Of course, as explained, this precludes the possibility of identifying LINERs. Note that we include column 5 from Table 8 in Table 11 (column 7), which together present multiwavelength nuclear activity diagnostics.

According to Table 8, emission-line ratio information exists for 22 galaxies in our sample. Out of these 22, 10 are classified as optSF (45.5\%), 2 are optTO/SF (9\%), 1 is optTO (4.5\%), 3 are optAGN (13.6\%), 1 is an optLINER/AGN (4.5\%), 1 is an opt-nonSF (4.5\%), 3 are optTO/LINER (13.6\%), and 1 is an optLINER $(4.5 \%)$. Thus, with the caveat of small-number statistics, we see that the clearest result of this classification is that star-forming systems represent the most numerous class, followed by AGNs. Apart from these, there appears to be only one clear LINER as well as a substantial number of mixed classifications.

\subsubsection{Optical Nuclear Excess}

Hubble Space Telescope (HST) data are available for a subset of our galaxy nuclei in seven HCGs. An observation log is given in Table 9. The high angular resolution $\left(\leqslant 00^{\prime} 1\right)$ of HST may provide a complementary means of identifying nuclear point sources. We identify these sources by examining the median divided image of each galaxy (we use a $13 \times 13$ pixel smoothing window and divide the original image by the smoothed one). Additionally, we use GALFIT (Peng et al. 2010) to fit surface brightness profiles and identify nuclear point sources. We compare the GALFIT-derived centers with the central sources 


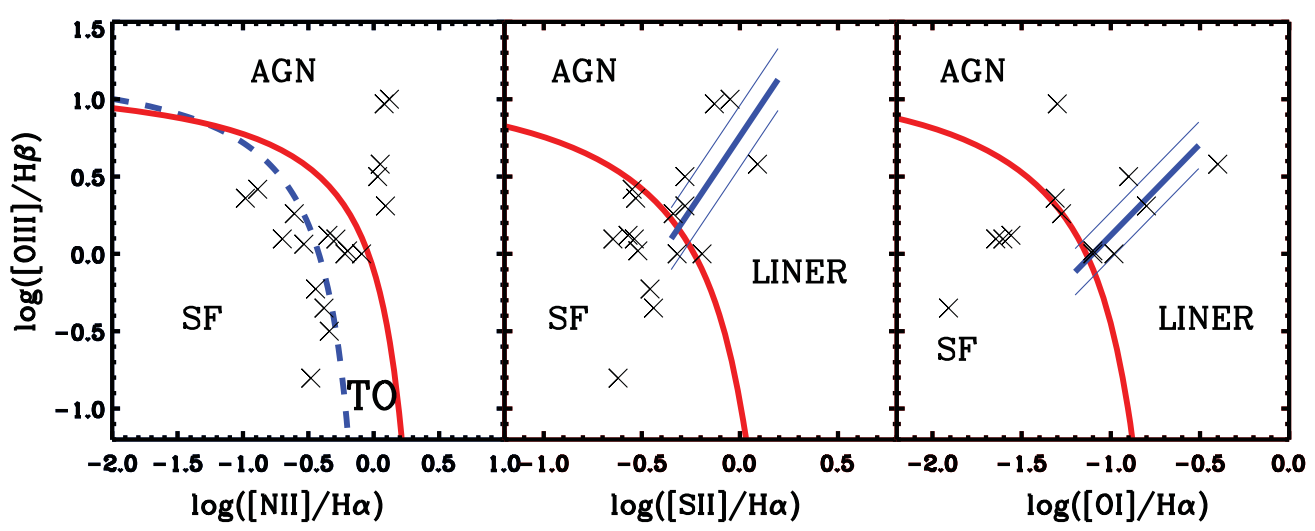

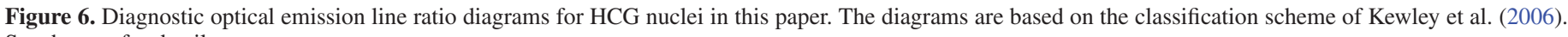
See the text for details.

(A color version of this figure is available in the online journal.)

Table 8

Nuclear Optical Spectroscopic Classification

\begin{tabular}{|c|c|c|c|c|}
\hline $\begin{array}{l}\text { ID } \\
(1)\end{array}$ & $\begin{array}{c}\text { K06(a) } \\
\text { (2) }\end{array}$ & $\begin{array}{c}\text { K06(b) } \\
\text { (3) }\end{array}$ & $\begin{array}{c}\mathrm{K} 06(\mathrm{c}) \\
(4)\end{array}$ & $\begin{array}{c}\text { Type } \\
(5)\end{array}$ \\
\hline $7 \mathrm{~A}$ & TO & SF & $\mathrm{SF}$ & $\mathrm{TO} / \mathrm{SF}$ \\
\hline $7 \mathrm{~B}$ & $\ldots$ & $\ldots$ & $\ldots$ & $\ldots$ \\
\hline $7 \mathrm{C}$ & SF & SF & $\ldots$ & SF \\
\hline 7D & SF & SF & $\ldots$ & $\mathrm{SF}$ \\
\hline $16 \mathrm{~A}$ & nonSF & LNR/AGN & LNR/AGN & LNR/AGN \\
\hline $16 \mathrm{~B}$ & nonSF & LNR & LNR & LNR \\
\hline $16 \mathrm{C}$ & TO & SF & SF & $\mathrm{TO} / \mathrm{SF}$ \\
\hline $16 \mathrm{D}$ & SF & SF & SF & SF \\
\hline $22 \mathrm{~A}$ & nonSF & AGN & $\mathrm{AGN}$ & $\mathrm{AGN}$ \\
\hline $22 \mathrm{~B}$ & $\ldots$ & $\ldots$ & $\ldots$ & $\ldots$ \\
\hline $22 \mathrm{C}$ & SF & $\ldots$ & $\ldots$ & SF \\
\hline 31ACE & SF & SF & SF & SF \\
\hline $31 \mathrm{~B}$ & SF & SF & SF & SF \\
\hline $31 \mathrm{~F}$ & $\ldots$ & $\ldots$ & $\ldots$ & $\ldots$ \\
\hline $31 \mathrm{G}$ & SF & SF & $\ldots$ & SF \\
\hline 31Q & $\mathrm{SF}$ & $\ldots$ & $\ldots$ & SF \\
\hline $42 \mathrm{~A}$ & nonSF & $\ldots$ & $\ldots$ & nonSF ${ }^{a}$ \\
\hline $42 B$ & $\ldots$ & $\ldots$ & $\ldots$ & $\ldots$ \\
\hline $42 \mathrm{C}$ & $\ldots$ & $\ldots$ & $\ldots$ & $\ldots$ \\
\hline $42 \mathrm{D}$ & $\ldots$ & $\ldots$ & $\ldots$ & $\ldots$ \\
\hline $59 \mathrm{~A}$ & TO & LNR & LNR & TO/LNR \\
\hline $59 B$ & TO & $\ldots$ & $\ldots$ & $\mathrm{TO}^{\mathrm{a}}$ \\
\hline $59 \mathrm{C}$ & SF & $\ldots$ & $\ldots$ & $\mathrm{SF}^{\mathrm{a}}$ \\
\hline 59D & SF & SF? & SF? & SF? \\
\hline $62 \mathrm{~A}$ & TO & $\mathrm{SF}$ & $\mathrm{LNR} / \mathrm{SF}$ & TO/LNR? \\
\hline $62 \mathrm{~B}$ & $\ldots$ & $\ldots$ & $\ldots$ & $\ldots$ \\
\hline $62 \mathrm{C}$ & $\ldots$ & $\ldots$ & $\ldots$ & $\ldots$ \\
\hline $62 \mathrm{D}$ & $\ldots$ & $\ldots$ & $\ldots$ & $\ldots$ \\
\hline $90 \mathrm{~A}$ & nonSF & $\mathrm{AGN}$ & AGN & $\mathrm{AGN}$ \\
\hline $90 \mathrm{~B}$ & $\ldots$ & $\ldots$ & $\ldots$ & $\ldots$ \\
\hline $90 \mathrm{C}$ & $\ldots$ & $\ldots$ & $\ldots$ & $\ldots$ \\
\hline $90 \mathrm{D}$ & TO & SF & LNR? & TO/LNR? \\
\hline $92 \mathrm{~B}$ & $\ldots$ & $\ldots$ & $\ldots$ & $\ldots$ \\
\hline $92 \mathrm{C}$ & nonSF & $\mathrm{AGN}$ & $\ldots$ & AGN \\
\hline $92 \mathrm{D}$ & $\ldots$ & $\ldots$ & $\ldots$ & $\ldots$ \\
\hline $92 \mathrm{E}$ & $\ldots$ & $\ldots$ & $\ldots$ & $\ldots$ \\
\hline $92 \mathrm{~F}$ & $\ldots$ & $\ldots$ & $\ldots$ & $\ldots$ \\
\hline
\end{tabular}

Notes. Columns give nuclear classification results based on K06 emission line ratio diagrams as follows: (1) $[\mathrm{O} \mathrm{III}] / \mathrm{H} \beta$ vs. $[\mathrm{N} \mathrm{II}] / \mathrm{H} \alpha$ (K06-a); (2) [O III] $/ \mathrm{H} \beta$ vs. [S II] $/ \mathrm{H} \alpha$ (K06-b); (3) [O III] $/ \mathrm{H} \beta$ vs. [O I] $/ \mathrm{H} \alpha$ (K06-c). Nuclear activity classifications are TO (transition object), SF (star forming), LNR (LINER), AGN (supermassive black hole accretion), and nonSF (either LNR or AGN).

a Based on $[\mathrm{N} \mathrm{II}] / \mathrm{H} \alpha$ only. detected in median divided images. The findings are summarized in columns 10 and 11 of Table 11. A " $y$ " in column 10 indicates that a nuclear point source in the median-divided image is detected, while a " $y$ " in column 11 indicates that the source coincides with the GALFIT center, within the positional uncertainties ( 3 pixels). It turns out that, due to the mostly disturbed nature of these galaxies, GALFIT is unable to either converge or provide good fits. We discuss this issue further later in the paper (Section 5.4).

\subsection{Radio}

Radio detections of nuclei constitute possible complementary evidence of AGN activity (in the case of radio-loud AGNs). Conversely, in star-forming galaxies there is a well-known correlation between the $1.4 \mathrm{GHz}(21 \mathrm{~cm})$ luminosity density, $L_{v 1.4}$, and SFR (e.g., Bell 2003). Thus, if the SFR is known, one can use this correlation to obtain an estimate for the $L_{v 1.4}$, as well as the $1.4 \mathrm{GHz}$ flux density, $f_{v 1.4, \text { est }}$. Any significant excess between the observed flux density, $f_{v 1.4, \text { obs }}$, and $f_{v 1.4, \text { est }}$ may be an indication of AGN activity.

We searched the archives of all publicly available major radio surveys, and found detections for 12 of our galaxies in 4 of the surveys. Details on the radio detections are given in Table 10. As none of the catalogs has full sky coverage, a non-detection does not necessarily imply the lack of radio emission. The catalogs also vary in sensitivity, resolution, and positional accuracy. We use both the NVSS and FIRST detections, together with UV+IR SFR values of Tzanavaris et al. (2010) to estimate the $f_{v 1.4 \text {,est }}$ by means of the Bell (2003) correlation. This correlation has two different forms, depending on whether a galaxy has $M_{V}>-21$ or not. We estimated $M_{V}<-21$ for all of our galaxies by using the $B$ and $R$-band values for HCG galaxies in Hickson et al. (1989) and the color transformations of Fukugita et al. (1995). Using the Bell (2003) correlation, we thus calculated $f_{v 1.4, \text { est }}$. We plot $f_{v 1.4, \text { est }}$ against $f_{v 1.4 \text {,obs }}$ in Figure 7 for the NVSS and FIRST detected galaxies. For 9 out of 12 galaxies with radio detections the estimated and observed flux density appear to be consistent with each other. The absence of a radio excess for these nine galaxies is indicated by "n" in column 9 of Table 11. We note though that for galaxies HCG $16 \mathrm{~A}, 90 \mathrm{~A}$, and $62 \mathrm{~A}$, there is an indication of an excess in $f_{v 1.4, \text { obs }}$ (" $\mathrm{y}$ " in column 9 of Table 11). For the first two this is entirely consistent with their AGN classification in the optical. In addition, HCG 90 A has high X-ray luminosity, further consistent with AGN activity. Our optical classification for the third is LINER, and the radio 
Table 9

HST Data for Galaxies in This Paper

\begin{tabular}{|c|c|c|c|c|c|c|c|}
\hline (1) & Galaxy & $\begin{array}{c}H S T \\
\text { Instrument } \\
\text { (3) }\end{array}$ & (4) & $\begin{array}{c}\text { Exp. Time } \\
\text { (s) } \\
\text { (5) }\end{array}$ & (6) & $\begin{array}{c}\text { Program } \\
\text { ID } \\
\text { (7) }\end{array}$ & $\begin{array}{l}\text { PI } \\
(8)\end{array}$ \\
\hline \multirow[t]{2}{*}{ HCG 07} & A, B, D & $\mathrm{ACS} / \mathrm{WFC}$ & F606W & 1230 & 2006 Sep & 10787 & Charlton \\
\hline & C & ACS/WFC & F606W & 1230 & 2006 Sep & & \\
\hline \multirow[t]{4}{*}{ HCG 16} & A & WFPC2 & F606W & 1900 & $2007 \mathrm{Jul}$ & & \\
\hline & $\mathrm{C}$ & WFPC2 & F606W & 1900 & 2007 Aug & & \\
\hline & $\mathrm{D}$ & WFPC2 & F606W & 1900 & 2007 Aug & & \\
\hline & A, B & WFPC2 & F606W & 1900 & $2007 \mathrm{Sep}$ & & \\
\hline \multirow[t]{3}{*}{ HCG 22} & B & WFPC2 & F606W & 1900 & 2007 Aug & & \\
\hline & $\mathrm{C}$ & WFPC2 & F606W & 1900 & 2007 Sep & & \\
\hline & A & WFPC2 & F606W & 1900 & 2007 Sep & & \\
\hline HCG 31 & $\mathrm{~A}-\mathrm{C}, \mathrm{E}-\mathrm{H}$ & ACS/WFC & F606W & 1230 & $2006 \mathrm{Aug}$ & & \\
\hline \multirow[t]{3}{*}{$\mathrm{HCG} 42$} & B & WFPC2 & F606W & 4200 & 2007 Dec & & \\
\hline & $\mathrm{D}$ & WFPC2 & F606W & 4200 & $2007 \mathrm{Dec}$ & & \\
\hline & $\mathrm{A}, \mathrm{C}$ & $\mathrm{ACS} / \mathrm{WFC}$ & F606W & 1230 & 2007 Dec & & \\
\hline \multirow[t]{2}{*}{ HCG 59} & A, C, D & ACS/WFC & F606W & 1230 & 2006 Nov & & \\
\hline & B, I & $\mathrm{ACS} / \mathrm{WFC}$ & F606W & 1230 & 2006 Nov & & \\
\hline HCG 62 & & & & No HST data & & & \\
\hline HCG 90 & & & & No HST data & & & \\
\hline \multirow[t]{3}{*}{ HCG 92} & $\mathrm{~B}, \mathrm{D}$ & WFC3 & F606W & 1395 & 2009 Aug & 11502 & Noll \\
\hline & $\mathrm{C}, \mathrm{B}$ & WFC3 & F606W & 1395 & 2009 Aug & & \\
\hline & E & WFC3 & F606W & 1395 & $2009 \mathrm{Jul}$ & & \\
\hline
\end{tabular}

Notes. Columns give: 1: compact group ID; 2: galaxies observed; 3: instrument used; 4: filter; 5: exposure time; 6: observation date; 7: program ID; 8: program PI.

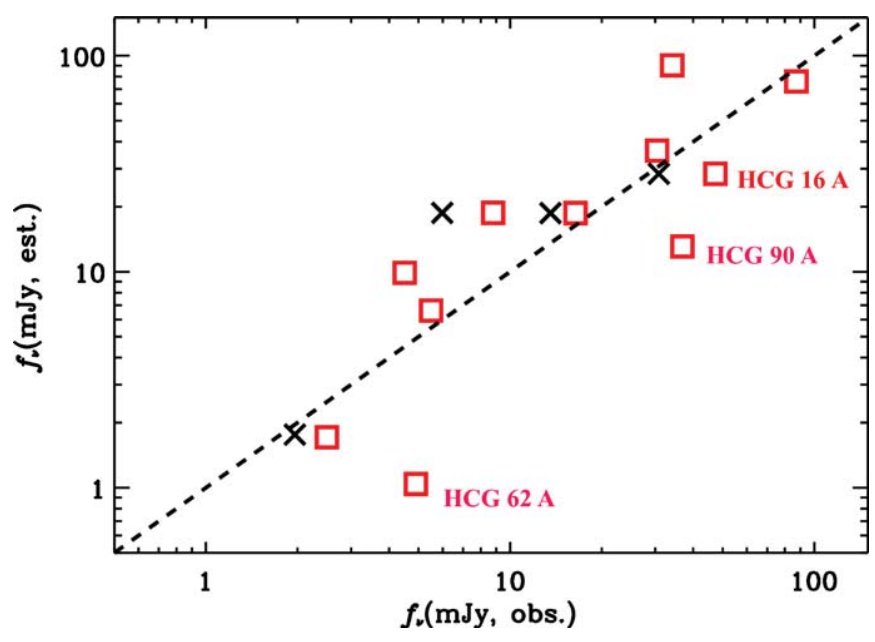

Figure 7. 1.4 GHz flux density, estimated from the radio-SFR correlation of Bell (2003), against observed 1.4 GHz flux density. Red squares correspond to NVSS and black crosses to FIRST data (see Table 7). The dashed line is the locus of equal estimated and observed flux densities. The three labeled HCG galaxies that show an excess in observed flux density are all known AGNs.

(A color version of this figure is available in the online journal.)

excess may suggest that it also hosts an AGN (recall that, as mentioned, a majority of LINERs do harbor AGNs). In spite of this consistency, note that for these galaxies $f_{v 1.4 \text {,est }}$ is still within a factor of $\sim 2$ from the corresponding $f_{\nu 1.4 \text {,obs }}$ value. This factor also corresponds to the uncertainty of the radio-SFR correlation, so this should not be considered a robust result. Unfortunately, we are unable to comment on galaxies for which we do not have any other classification, as none of these are detected in the radio.

We also compare our combined X-ray-radio results for HCG nuclei with the work of Ranalli et al. (2003, 2012) for entire galaxies. Following Ranalli et al. (2012), in Figure 8 we plot soft

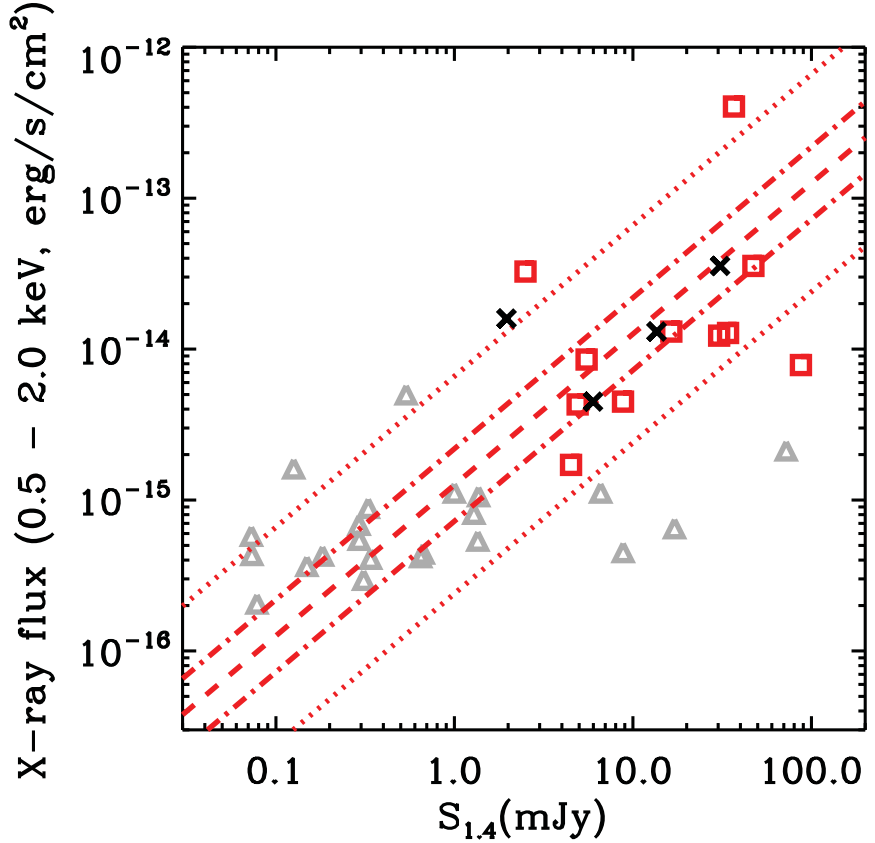

Figure 8. Radio vs. X-ray soft band fluxes for HCG nuclei in this paper. HCG radio fluxes are shown as crosses (FIRST) or red squares (NVSS). Grey triangles are VLA-COSMOS galaxies classified as star-forming by Ranalli et al. (2012, "c1" class from their Tables 1 and 2.). The red dashed line is the radio-X-ray correlation of Ranalli et al. (2003, their Equation (8) converted to flux using the average distance of our nine HCG nuclei.)

(A color version of this figure is available in the online journal.)

band X-ray luminosities against 1.4 GHz fluxes from NVSS and FIRST (red squares and crosses, respectively) for HCG nuclei. We also show the observational X-ray-radio correlation for local star-forming galaxies established by Ranalli et al. (2003) converted to flux using the mean distance of the HCG nuclei. For 
Table 10

Radio Detections of HCG Nuclei in This Sample

\begin{tabular}{|c|c|c|c|c|c|c|c|c|c|c|c|c|c|c|c|}
\hline \multirow[b]{2}{*}{ HCG ID } & \multicolumn{5}{|c|}{ FIRST $^{\mathrm{a}}$} & \multicolumn{3}{|c|}{$\mathrm{NVSS}^{\mathrm{b}}$} & \multicolumn{3}{|c|}{ SUMMS $^{c}$} & \multicolumn{4}{|c|}{$\mathrm{WISH}^{\mathrm{d}}$} \\
\hline & (2) & $\begin{array}{c}f_{20, \mathrm{p}} \\
(\mathrm{mJy}) \\
(3)\end{array}$ & $\begin{array}{c} \pm \\
(\mathrm{mJy}) \\
(4)\end{array}$ & $\begin{array}{c}f_{20, \mathrm{i}} \\
(\mathrm{mJy}) \\
(5)\end{array}$ & $\begin{array}{c}\Delta \text { pos } \\
\left({ }^{\prime \prime}\right) \\
(6)\end{array}$ & $\begin{array}{c}f_{20, \mathrm{i}} \\
(\mathrm{mJy}) \\
(7)\end{array}$ & $\begin{array}{c} \pm \\
(\mathrm{mJy}) \\
(8)\end{array}$ & $\begin{array}{c}\Delta \operatorname{pos} \\
\left({ }^{\prime \prime}\right) \\
(9)\end{array}$ & $\begin{array}{c}f_{36, \mathrm{i}} \\
(\mathrm{mJy}) \\
(10)\end{array}$ & $\begin{array}{c} \pm \\
(\mathrm{mJy}) \\
(11)\end{array}$ & $\begin{array}{c}\Delta \text { pos } \\
\left({ }^{\prime \prime}\right) \\
(12)\end{array}$ & $\begin{array}{c}f_{352, \mathrm{p}} \\
(\mathrm{mJy}) \\
(13)\end{array}$ & $\begin{array}{c}f_{352, \mathrm{i}} \\
(\mathrm{mJy}) \\
(14)\end{array}$ & $\begin{array}{c} \pm \\
(\mathrm{mJy}) \\
(15)\end{array}$ & $\begin{array}{c}\Delta \mathrm{pos} \\
\left({ }^{\prime \prime}\right) \\
(16)\end{array}$ \\
\hline 7A & 0.014 & 7.43 & 0.131 & 13.57 & 0.84 & 16.4 & 0.7 & 1.86 & $\ldots$ & $\ldots$ & $\ldots$ & $\ldots$ & $\ldots$ & $\ldots$ & $\ldots$ \\
\hline $7 \mathrm{C}$ & $\ldots$ & $\ldots$ & $\ldots$ & $\ldots$ & $\ldots$ & 4.5 & 0.5 & 8.04 & $\ldots$ & $\ldots$ & $\ldots$ & $\ldots$ & $\ldots$ & $\ldots$ & $\ldots$ \\
\hline $16 \mathrm{~A}$ & 0.014 & 12.80 & 0.159 & 30.81 & 2.52 & 47.3 & 2.1 & 1.98 & $\ldots$ & $\ldots$ & $\ldots$ & 93 & 100 & 4.6 & 4.5 \\
\hline $16 \mathrm{~B}$ & 0.014 & 1.98 & 0.158 & 1.96 & 0.6 & $\ldots$ & $\ldots$ & $\ldots$ & $\ldots$ & $\ldots$ & $\ldots$ & 51 & 53 & 4.8 & 0.54 \\
\hline $16 \mathrm{C}$ & $\ldots$ & $\ldots$ & $\ldots$ & $\ldots$ & $\ldots$ & 87.3 & 3.2 & 2.04 & $\ldots$ & $\ldots$ & $\ldots$ & $\ldots$ & $\ldots$ & $\ldots$ & $\ldots$ \\
\hline $16 \mathrm{D}$ & $\ldots$ & $\ldots$ & $\ldots$ & $\ldots$ & $\ldots$ & 34.1 & 1.1 & 0.78 & $\ldots$ & $\ldots$ & $\ldots$ & $\ldots$ & $\ldots$ & $\ldots$ & $\ldots$ \\
\hline 31ACE & $\ldots$ & $\ldots$ & $\ldots$ & $\ldots$ & $\ldots$ & 30.4 & 1.6 & 6.9 & $\ldots$ & $\ldots$ & $\ldots$ & $\ldots$ & $\ldots$ & $\ldots$ & $\ldots$ \\
\hline $31 \mathrm{G}$ & $\ldots$ & $\ldots$ & $\ldots$ & $\ldots$ & $\ldots$ & 5.5 & 0.5 & 4.14 & $\ldots$ & $\ldots$ & $\ldots$ & $\ldots$ & $\ldots$ & $\ldots$ & $\ldots$ \\
\hline $42 \mathrm{~A}$ & $\ldots$ & $\ldots$ & $\ldots$ & $\ldots$ & $\ldots$ & 2.5 & 0.5 & 9 & $\ldots$ & $\ldots$ & $\ldots$ & $\ldots$ & $\ldots$ & $\ldots$ & $\ldots$ \\
\hline $59 \mathrm{~A}$ & 0.014 & 6.18 & 0.187 & 5.98 & 1.44 & 8.8 & 1.0 & 8.4 & $\ldots$ & $\ldots$ & $\ldots$ & $\ldots$ & $\ldots$ & $\ldots$ & $\ldots$ \\
\hline $62 \mathrm{~A}$ & $\ldots$ & $\ldots$ & $\ldots$ & $\ldots$ & $\ldots$ & 4.9 & 0.5 & 6.78 & $\ldots$ & $\ldots$ & $\ldots$ & $\ldots$ & $\ldots$ & $\ldots$ & $\ldots$ \\
\hline $90 \mathrm{~A}$ & $\ldots$ & $\ldots$ & $\ldots$ & $\ldots$ & $\ldots$ & 36.8 & 1.5 & 3.54 & 45.6 & 1.7 & 6.6 & $\ldots$ & $\ldots$ & $\ldots$ & $\ldots$ \\
\hline
\end{tabular}

Notes. Columns are: 1: HCG galaxy ID; 2: FIRST probability that the source is spurious; 3: FIRST peak flux at 20 cm; 4: FIRST error on peak flux; 5: FIRST integrated $20 \mathrm{~cm}$ flux; 6: FIRST positional uncertainty in arcseconds; 7: NVSS integrated $20 \mathrm{~cm}$ flux; 8: NVSS flux error; 9: NVSS positional uncertainty in arcseconds; 10: SUMMS integrated $36 \mathrm{~cm}$ flux; 11: SUMMS flux error; 12: SUMMS positional uncertainty in arcseconds; 13: WISH 352 MHz peak flux; 14: WISH $352 \mathrm{MHz}$ integrated flux; 15: WISH flux error; 16: WISH positional uncertainty in arcseconds.

${ }^{a}$ Faint Images of the Radio Sky at Twenty-cm survey (Becker et al. 1995).

b NRAO-VLA Sky Survey (Condon et al. 1998).

c Sydney University Molonglo Sky Survey (Bock et al. 1999).

${ }^{\mathrm{d}}$ Westerbork in the Southern Hemisphere survey (De Breuck et al. 2002).

comparison, we further plot VLA COSMOS sources detected in C-COSMOS and classified as star-forming by Ranalli et al. (2012, class "c1," their Tables 1 and 2). In this figure, HCG nuclei appear to blend smoothly with the more distant lowerflux COSMOS star-forming sources, although with considerable scatter. The topic of X-ray emission in HCGs as a function of star formation rate will be examined in greater detail in a forthcoming publication.

\section{3. $X-R a y-U V$}

Unlike in the X-rays, each galaxy's nucleus is well defined in the UV regime as an emission peak in the central region of the galaxy. To identify nuclear X-ray point sources for each galaxy we thus visually inspect all X-ray point sources for spatial coincidence with the central intensity peak of UV sources at $2600 \AA$. Specifically, we examine the degree of overlap between the AE-determined Chandra PSF and a UVOT "PSF," defined as a circular region 3 " in diameter centered at the $u v w 1$ intensity peak. Unlike the case for photometry, the choice of UV circular region size here is solely determined by the $u v w 1$ PSF FWHM of 2".37. Note that the absolute astrometry for both UVOT and Chandra is very good, as measured relative to the International Celestrial Reference System (Ma \& Feissel 1998). We estimate that in the worst case scenario this could lead to a maximum spurious offset between two coincident sources of $\sim 0^{\prime \prime} .7$ (combining in quadrature $\sim 0^{\prime \prime} 4$ from UVOT, Breeveld et al. (2010), and $\sim 0$ '. 6 from Chandra, http://cxc.harvard.edu/cal/ASPECT/celmon/).

We also calculate the offset, $\Delta \theta$, in arcseconds between the $\mathrm{X}$-ray and the UV galaxy nucleus as determined by the AE center and UV peak emission, respectively. This is shown in column 3 of Table 11, while column 4 reports whether we consider that $\mathrm{X}$-ray-UV nuclear counterparts exist for a given galaxy based on an overlap between the X-ray and UV PSFs. Due to the different resolution between the UV and the X-rays, our primary criterion for the existence of counterparts is the PSF overlap rather than $\Delta \theta$. We do note that all offsets are less than $\sim 3^{\prime \prime}$. If the PSF overlap criterion indicates that X-ray -UV counterparts exist, this is indicated by " $y$ " in column 4 . There are 22 such cases, which are best candidates for X-ray-UV counterparts. This reduces the original number of 27 nuclear X-ray sources estimated without strict use of the PSF overlap criterion by 5 . Conversely, an "n" indicates no counterparts (10 cases). In two cases (22A, 31ACE) it is not clear which X-ray source is the best counterpart, as two X-ray PSFs overlap with the UV PSF. This is indicated by an " $\mathrm{m}$ " (for "multiple") in column 3. A question mark indicates that the X-ray-UV counterpart is uncertain, as described in Section 5.4 (three cases).

To quantify the relative contributions coming from the $\mathrm{X}$-rays and the UV we calculate the $\alpha_{\mathrm{OX}}$ index defined above and tabulate our results in columns 5 and 6 of Table 11, corrected and uncorrected for intrinsic extinction, respectively. The mean $\alpha_{\mathrm{OX}}$ values and $1 \sigma$ standard deviations are $\alpha_{\mathrm{OX}}=-2.33 \pm 0.31$ (corrected) and $\alpha_{\mathrm{OX}}=-2.17 \pm 0.32$ (uncorrected). Thus, overall, the extinction correction does not significantly affect $\alpha_{\text {OX }}$ values. We plot $\alpha_{\text {OX }}$ versus $2600 \AA$ luminosity, $v L_{\nu 2600}$, in Figure 9. The well-known correlation for 333 moderate- to high-luminosity AGNs by Steffen et al. (2006, hereafter S06) is shown by the dashed line and the $\pm 3 \sigma$ scatter of the data points on the correlation by the solid lines. The S06 AGNs are shown as black (magenta for upper limits) stars (their tables 1 and 2). We also show the AGNs of Lusso et al. (2010, herafter LC10) as cyan dots. The black open circles are the LINERs of Eracleous et al. (2010b). Our HCG nuclei are shown in color, according to their optical spectroscopic classification (Section 5.1.1), or as black crosses if there is no such classification. HCG upper limits are shown by downward pointing arrows. In the interest of clarity, in this and subsequent plots mixed classifications (Table 8, column 5, and Table 11, column 7) have been simplified as follows: AGN? $\rightarrow$ AGN; $\mathrm{SF} ? \rightarrow \mathrm{SF} ; \mathrm{TO} / \mathrm{AGN}, \mathrm{TO} / \mathrm{SF}, \mathrm{TO} / \mathrm{LNR} \rightarrow \mathrm{TO} ; \mathrm{LNR} / \mathrm{AGN} \rightarrow$ 
Table 11

Multiwavelength Analysis of HCG Nuclear Sources

\begin{tabular}{|c|c|c|c|c|c|c|c|c|c|c|}
\hline \multirow{2}{*}{$\begin{array}{l}\text { HCG ID } \\
\text { (1) }\end{array}$} & \multirow{2}{*}{$\begin{array}{c}\text { Morphology } \\
\text { (2) }\end{array}$} & \multirow{2}{*}{$\begin{array}{l}\Delta \theta \\
(3)\end{array}$} & \multirow{2}{*}{$\begin{array}{c}\text { X-ray-UV } \\
\text { Counterparts } \\
\text { (4) }\end{array}$} & \multirow{2}{*}{$\begin{array}{c}\alpha_{\mathrm{OX}} \\
\text { (Corrected) } \\
(5)\end{array}$} & \multirow{2}{*}{$\begin{array}{c}\alpha_{\mathrm{OX}} \\
\text { (Uncorrected) } \\
(6)\end{array}$} & \multirow{2}{*}{$\begin{array}{l}\text { Optical } \\
\text { Type } \\
(7)\end{array}$} & \multirow{2}{*}{$\begin{array}{c}\text { X-Ray } \\
\text { Strong AGN } \\
\text { (8) }\end{array}$} & \multirow{2}{*}{$\begin{array}{c}\text { Radio } \\
\text { Excess } \\
(9)\end{array}$} & \multicolumn{2}{|c|}{$\begin{array}{c}\text { Optical Nuclear } \\
\text { Source }\end{array}$} \\
\hline & & & & & & & & & $(10)$ & (11) \\
\hline $7 \mathrm{~A}$ & $\mathrm{Sb}$ & 1.2 & $\mathrm{y}$ & -2.23 & -1.96 & $\mathrm{TO} / \mathrm{SF}$ & $\mathrm{n}$ & $\mathrm{n}$ & $\mathrm{y}$ & $\mathrm{y}$ \\
\hline $7 \mathrm{~B}$ & SB0 & 1.1 & $\mathrm{y}$ & -2.31 & -2.26 & $\ldots$ & $\mathrm{n}$ & $\ldots$ & $\mathrm{y}$ & $\mathrm{y}$ \\
\hline $7 \mathrm{C}$ & $\mathrm{SBc}$ & 0.5 & $\mathrm{y}$ & -2.39 & -2.30 & SF & $\mathrm{n}$ & $\mathrm{n}$ & $\mathrm{y}$ & $\mathrm{n}$ \\
\hline $7 \mathrm{D}$ & $\mathrm{SBc}$ & $\ldots$ & $\mathrm{n}$ & -2.51 & -2.46 & $\mathrm{SF}$ & $\mathrm{n}$ & $\ldots$ & $\mathrm{y}$ & $\mathrm{y}$ \\
\hline $16 \mathrm{~A}$ & SBab & 3.0 & $\mathrm{y}$ & -2.16 & -1.92 & LINER/AGN & $\mathrm{n}$ & $\mathrm{y}$ & $\mathrm{y}$ & $\ldots$ \\
\hline $16 \mathrm{~B}$ & $\mathrm{Sab}$ & 0.8 & $\mathrm{y}$ & -1.68 & -1.57 & LINER & $\mathrm{y}$ & $\mathrm{n}$ & $\mathrm{y}$ & $\ldots$ \\
\hline $16 \mathrm{C}$ & $\operatorname{Im}$ & 3.0 & $\mathrm{y}$ & -2.75 & -2.35 & $\mathrm{TO} / \mathrm{SF}$ & $\mathrm{n}$ & $\mathrm{n}$ & $\mathrm{y}$ & $\ldots$ \\
\hline $16 \mathrm{D}$ & $\operatorname{Im}$ & 1.1 & $\mathrm{y}$ & -2.61 & -1.96 & SF & $\mathrm{n}$ & $\mathrm{n}$ & $\mathrm{y}$ & $\ldots$ \\
\hline $22 \mathrm{~A}$ & E2 & $0.8,0.7$ & $\mathrm{ym}$ & -2.35 & -2.33 & AGN & $\mathrm{n}$ & $\ldots$ & $\mathrm{y}$ & $\ldots$ \\
\hline $22 \mathrm{~B}$ & $\mathrm{Sa}$ & 1.7 & $\mathrm{y}$ & -2.13 & -2.11 & $\ldots$ & $\mathrm{n}$ & $\ldots$ & $\mathrm{y}$ & $\ldots$ \\
\hline $22 \mathrm{C}$ & SBcd & $\ldots$ & $\mathrm{n}$ & -2.42 & -2.36 & SF & $\mathrm{n}$ & $\ldots$ & $\mathrm{n}$ & $\ldots$ \\
\hline 31ACE & $\mathrm{Sdm}$ & $1.7,0.7$ & $\mathrm{ym}$ & -2.50 & -2.34 & SF & $\mathrm{n}$ & $\mathrm{n}$ & $\mathrm{n}$ & $\ldots$ \\
\hline $31 \mathrm{~B}$ & $\mathrm{Sm}$ & $\ldots$ & $\mathrm{n}$ & -2.74 & -2.69 & SF & $\mathrm{n}$ & $\ldots$ & $\mathrm{n}$ & $\ldots$ \\
\hline $31 \mathrm{~F}$ & $\operatorname{Im}$ & $\ldots$ & $\mathrm{n}$ & -2.57 & -2.56 & $\ldots$ & $\mathrm{n}$ & $\ldots$ & $\mathrm{n}$ & $\ldots$ \\
\hline $31 \mathrm{G}$ & $\operatorname{Im}$ & 0.8 & $\mathrm{y}$ & -2.30 & -2.23 & SF & $\mathrm{n}$ & $\mathrm{n}$ & $\mathrm{y}$ & $\ldots$ \\
\hline $31 \mathrm{Q}$ & $\operatorname{Im}$ & 2.3 & $\mathrm{n} ?$ & -2.35 & -2.32 & SF & $\mathrm{n}$ & $\ldots$ & $\mathrm{n}$ & $\ldots$ \\
\hline $42 \mathrm{~A}$ & E3 & 1.8 & $\mathrm{y}$ & -2.05 & -2.01 & nonSF & $\mathrm{n}$ & $\mathrm{n}$ & $\mathrm{y}$ & $\mathrm{y}$ \\
\hline $42 B$ & SB0 & $\ldots$ & $\mathrm{n}$ & -2.32 & -2.28 & $\ldots$ & $\mathrm{n}$ & $\ldots$ & $\mathrm{y}$ & $\ldots$ \\
\hline $42 \mathrm{C}$ & E2 & 1.0 & $\mathrm{y}$ & -2.16 & -2.14 & $\ldots$ & $\mathrm{n}$ & $\ldots$ & $\mathrm{y}$ & $\ldots$ \\
\hline $42 \mathrm{D}$ & E2 & $\ldots$ & $\mathrm{n}$ & -2.12 & -2.09 & $\ldots$ & $\mathrm{n}$ & $\ldots$ & $\mathrm{y}$ & $\ldots$ \\
\hline $59 \mathrm{~A}$ & $\mathrm{Sa}$ & 1.8 & $\mathrm{y}$ & -2.47 & -1.90 & TO/LINER & $\mathrm{n}$ & $\mathrm{n}$ & $\mathrm{y}$ & $\mathrm{n}$ \\
\hline $59 \mathrm{~B}$ & E0 & 2.0 & $\mathrm{y} ?$ & -2.01 & -1.98 & TO & $\mathrm{n}$ & $\cdots$ & $\mathrm{y}$ & $\mathrm{y}$ \\
\hline $59 \mathrm{C}$ & $\mathrm{Sc}$ & $\ldots$ & $\mathrm{n}$ & -2.55 & -2.49 & $\mathrm{SF}$ & $\mathrm{n}$ & $\ldots$ & $\mathrm{y}$ & $\mathrm{y}$ \\
\hline 59D & $\operatorname{Im}$ & $\ldots$ & $\mathrm{n}$ & -2.63 & -2.58 & SF? & $\mathrm{n}$ & $\ldots$ & $\mathrm{n}$ & $\mathrm{n}$ \\
\hline $62 \mathrm{~A}$ & E3 & 1.1 & $\mathrm{y}$ & -2.16 & -2.14 & TO/LINER? & $\mathrm{n}$ & $\mathrm{y}$ & $\ldots$ & $\ldots$ \\
\hline $62 \mathrm{~B}$ & So & 0.1 & $\mathrm{y}$ & -2.16 & -2.15 & $\ldots$ & $\mathrm{n}$ & $\ldots$ & $\ldots$ & $\ldots$ \\
\hline $62 \mathrm{C}$ & S0 & 0.4 & $\mathrm{y}$ & -2.21 & -2.20 & $\ldots$ & $\mathrm{n}$ & $\ldots$ & $\ldots$ & $\ldots$ \\
\hline $62 \mathrm{D}$ & E2 & $\cdots$ & $\mathrm{n}$ & -2.17 & -2.08 & $\ldots$ & $\mathrm{n}$ & $\cdots$ & $\cdots$ & $\cdots$ \\
\hline $90 \mathrm{~A}$ & $\mathrm{Sa}$ & 3.3 & $\mathrm{y}$ & -1.31 & -1.01 & AGN & $\mathrm{y}$ & $\mathrm{y}$ & $\ldots$ & $\ldots$ \\
\hline $90 \mathrm{~B}$ & E0 & 0.4 & $\mathrm{y}$ & -2.49 & -2.18 & $\ldots$ & $\mathrm{n}$ & $\ldots$ & $\ldots$ & $\ldots$ \\
\hline $90 \mathrm{C}$ & E0 & 0.9 & $\mathrm{y}$ & -2.49 & -2.18 & $\ldots$ & $\mathrm{n}$ & $\ldots$ & $\ldots$ & $\ldots$ \\
\hline $90 \mathrm{D}$ & $\mathrm{Im}$ & $\ldots$ & $\mathrm{n}$ & -2.91 & -2.60 & TO/LINER? & $\mathrm{n}$ & $\ldots$ & $\ldots$ & $\ldots$ \\
\hline $92 \mathrm{~B}$ & $\mathrm{Sbc}$ & 0.6 & $\mathrm{y}$ & -2.68 & -2.37 & $\ldots$ & $\mathrm{n}$ & $\ldots$ & $\mathrm{y}$ & $\ldots$ \\
\hline $92 \mathrm{C}$ & Sbc & 0.9 & $\mathrm{y}$ & -1.81 & -1.51 & AGN & $\mathrm{y}$ & $\ldots$ & $\mathrm{y}$ & $\ldots$ \\
\hline $92 \mathrm{D}$ & $\mathrm{E} 2$ & 0.7 & $\mathrm{y}$ & -2.54 & -2.23 & $\ldots$ & $\mathrm{n}$ & $\ldots$ & $\mathrm{y}$ & $\cdots$ \\
\hline $92 \mathrm{E}$ & E1 & 1.5 & $\mathrm{y}$ & -2.57 & -2.27 & $\ldots$ & $\mathrm{n}$ & $\ldots$ & $\mathrm{y}$ & $\ldots$ \\
\hline $92 \mathrm{~F}$ & SAB0 & 2.1 & $\mathrm{y} ?$ & -2.49 & -2.19 & $\ldots$ & $\mathrm{n}$ & $\ldots$ & $\ldots$ & $\ldots$ \\
\hline
\end{tabular}

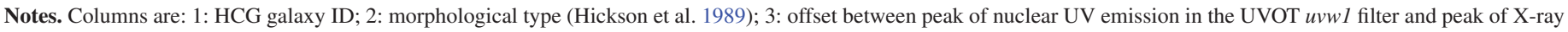

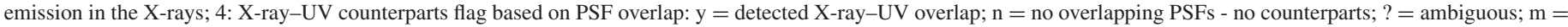

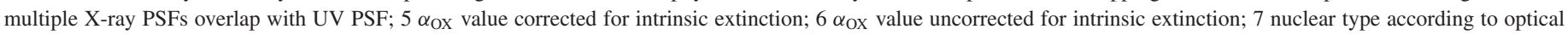

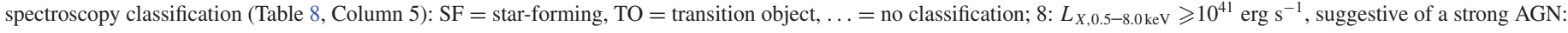

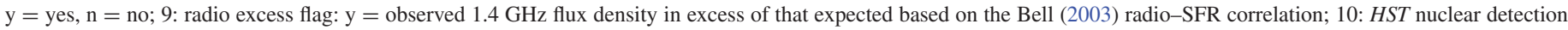

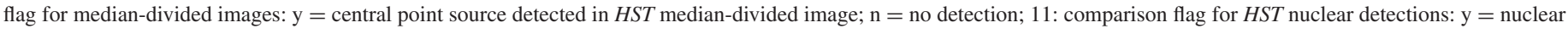
point source in HST median-divided image coincides with GALFIT center within 3 pixels; $\mathrm{n}=$ no coincidence within 3 pixels.

LNR. It can be seen that HCG nuclei do not occupy the same locus as strong AGNs and LINERs. This suggests that HCG nuclei are not likely to harbor strong AGNs or LINERs. To further explore whether this is also consistent with star formation being dominant in HCG nuclei, we make a comparison with star-forming galaxies from the compilation of Lehmer et al. (2010, hereafter L10). Those authors used multi-wavelength criteria to select star-forming galaxies among nearby $(<60 \mathrm{Mpc})$ luminous infrared galaxies (LIRGs). We select a sub-sample of 30 galaxies from this sample whose SFRs are in the range 0.011 to $17 M_{\odot} \mathrm{yr}^{-1}$, matching the SFR range of our HCG galaxies (Tzanavaris et al. 2010). Note that we do not need nuclear photometry of these galaxies in order to test our results. What we wish to investigate is whether the $\mathrm{X}$-ray and UV contributions in a galactic environment dominated by star formation are similar to our findings for the majority of
HCG nuclei. We thus calculate $\alpha_{\mathrm{OX}}$ for these systems and indicate them in Figure 9 as green crosses. The mean $\alpha_{\text {OX }}$ for these sources is $-1.90 \pm 0.33$ and the figure shows that there is significant overlap with our nuclei. In particular, the most relevant comparison is with HCG nuclei data not corrected for intrinsic extinction (right panel in Figure 9), as L10 did not carry out any such correction. At the same time, compared to strong AGNs, these galaxies seem to occupy a completely different region.

We quantify the comparisons between $\alpha_{\text {OX }}$ values for different data sets by carrying out a standard two-sided KS test, the results of which are shown in Figure 10. The probability that the $\alpha_{\mathrm{OX}}$ values for HCG nuclei come from the same distribution as the values for strong AGNs and LINERs is extremely small $\left(8 \times 10^{-28}\right.$ if corrected and $8 \times 10^{-27}$ if uncorrected for intrinsic extinction). In contrast, the probability that the $\alpha_{\mathrm{OX}}$ values for HCG nuclei uncorrected for intrinsic extinction come from the 

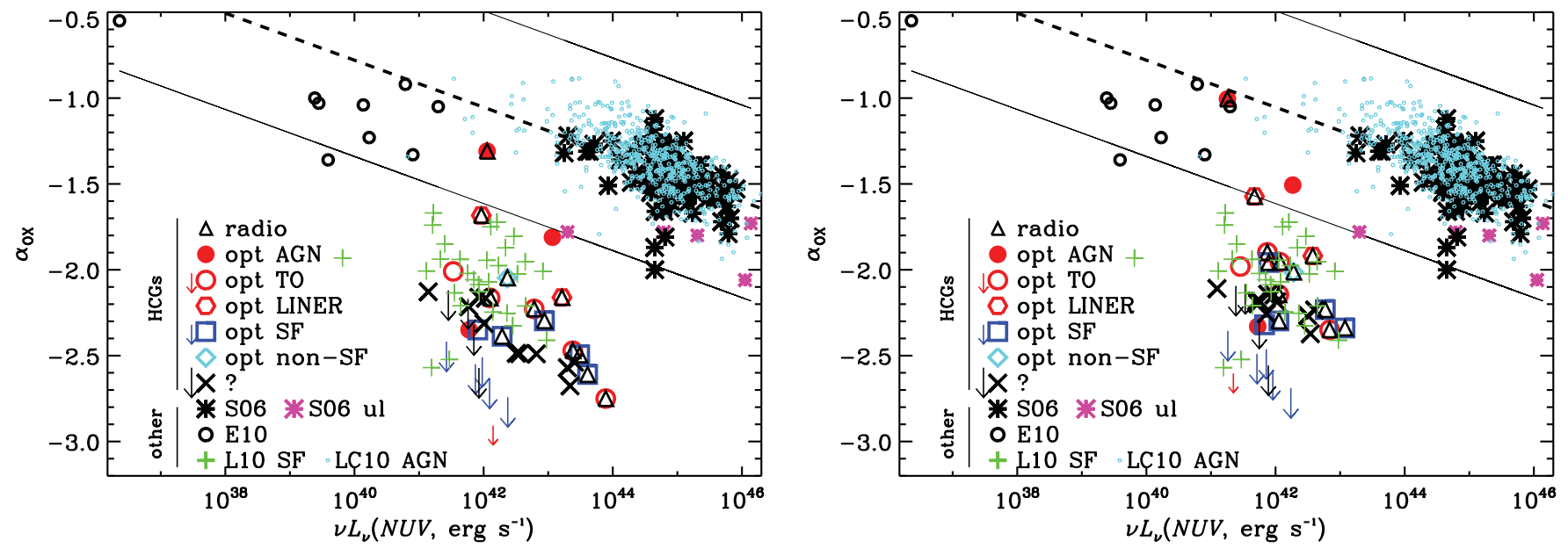

Figure 9. UV-to-X-ray spectral index, $\alpha_{\mathrm{OX}}$, vs. UV luminosity at $\sim 2600 \AA$. The left panel uses values corrected for intrinsic extinction, while the right panel uses uncorrected values. Symbols indicate optical nuclear classification (Section 5.1.1) as indicated in the legend: triangles, large open and filled circles, squares, diamonds, pentagons, and crosses are for HCG nuclei. Downward pointing arrows are upper limits for HCG nuclei based on upper limit X-ray flux estimates for $\mathrm{X}$-ray non-detections and are color-coded to indicate optical nuclear classifications (black if there are none). Note that in the interest of clarity, mixed classifications have been simplified, as explained in the text. Non-HCG data points are from Steffen et al. (2006, S06, ul = upper limits), Eracleous et al. (2010b, E10) Lehmer et al. (2010, L10), Lusso et al. (2010, LC10). The dashed line is the correlation for strong AGNs from S06. The two solid lines indicate the $\pm 3 \sigma$ region for AGNs (S06) and LINERs (E10). The green crosses are star-forming galaxies from L10, with SFRs in the same range as our HCG galaxies. These plots clearly suggest that most HCG nuclei do not follow the correlation for strong AGNs but are more similar to star-forming systems.

(A color version of this figure is available in the online journal.)

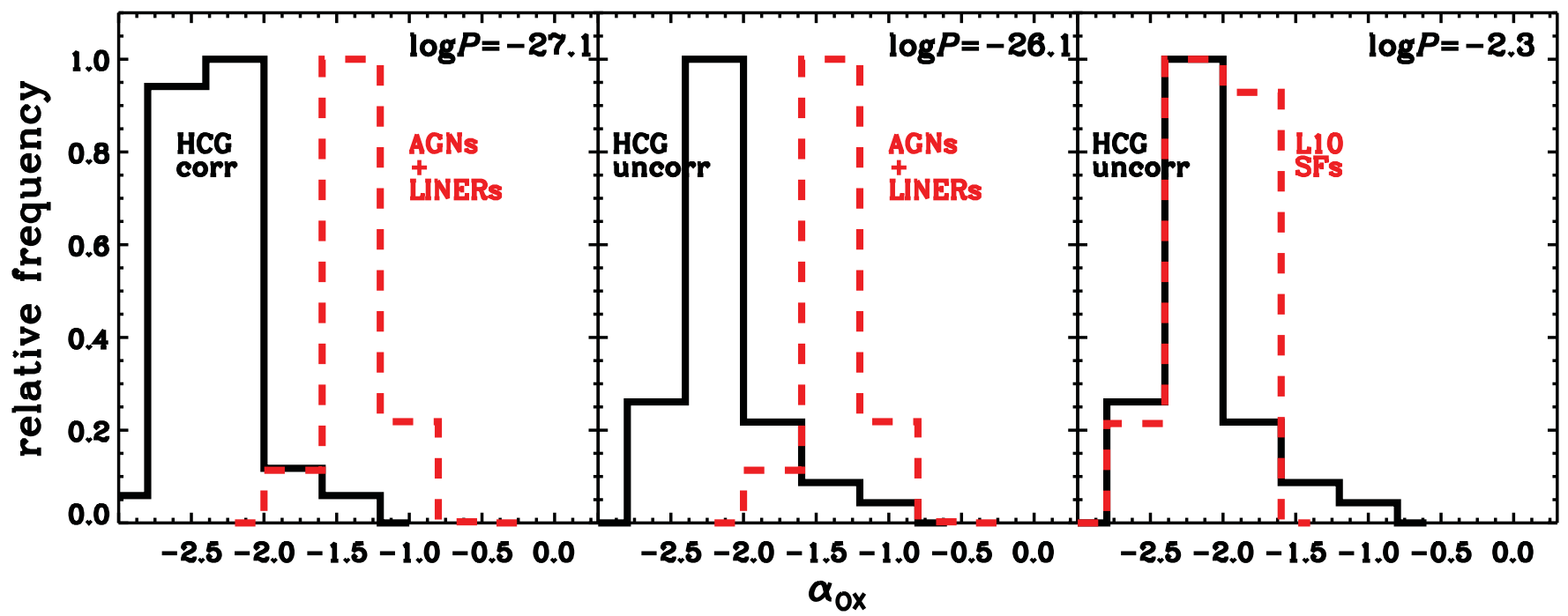

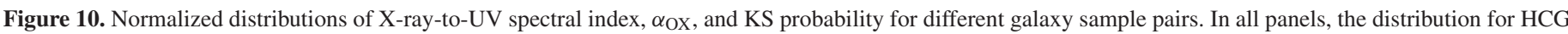

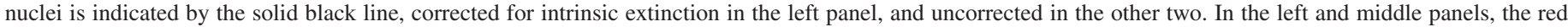

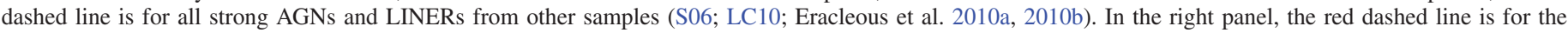

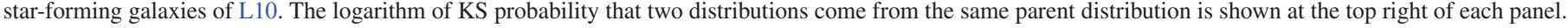

(A color version of this figure is available in the online journal.)

same distribution as those for the L10 star-forming galaxies is substantially larger at 0.005 . This number is not extremely large, as, after all, the L10 sample represents an extragalactic environment (LIRGs) distinct from compact groups. However, this probability may be high enough to suggest that HCG nuclei are at least more similar to star-forming galaxies than they are to strong AGNs. This is also evident from the overlap between the two distributions in the histogram of the rightmost panel in Figure 10. We thus conclude that, generally speaking, HCG nuclei do not follow the strong AGN correlation and are consistent with star formation being dominant.

Further, in Figure 9 there is a trend with increasing $v L_{v 2600}$ and decreasing $\alpha_{\mathrm{OX}}$ for nuclei to be both farther below the strong AGN locus defined by the correlation and to be optically classified as either SF or TO. To further investigate this possible connection between $\alpha_{\mathrm{OX}}$ and star formation, we plot $\alpha_{\mathrm{OX}}$ versus SSFR in the top row of Figure 11 using two different color and symbol schemes. In the left panel, symbols are coded to indicate the optically based nuclear activity classification (where available). In this panel, optSFs preferentially occupy the high-SSFR/low- $\alpha_{\mathrm{OX}}$ region of parameter space, where $\alpha_{\mathrm{OX}} \lesssim-2.3$. In contrast, optLINERs, opt-nonSFs, optAGNs, and some optTOs inhabit the low-SSFR/high- $\alpha_{\mathrm{OX}}$ region of parameter space. The fact that two opt-TOs are found in the optSF region is consistent with their definition, as one would expect transition objects to appear in both regions. If this apparent distribution pattern in $\alpha_{\mathrm{OX}}-\mathrm{SSFR}$ space is a real effect, then we predict that the nuclei for which there is no 

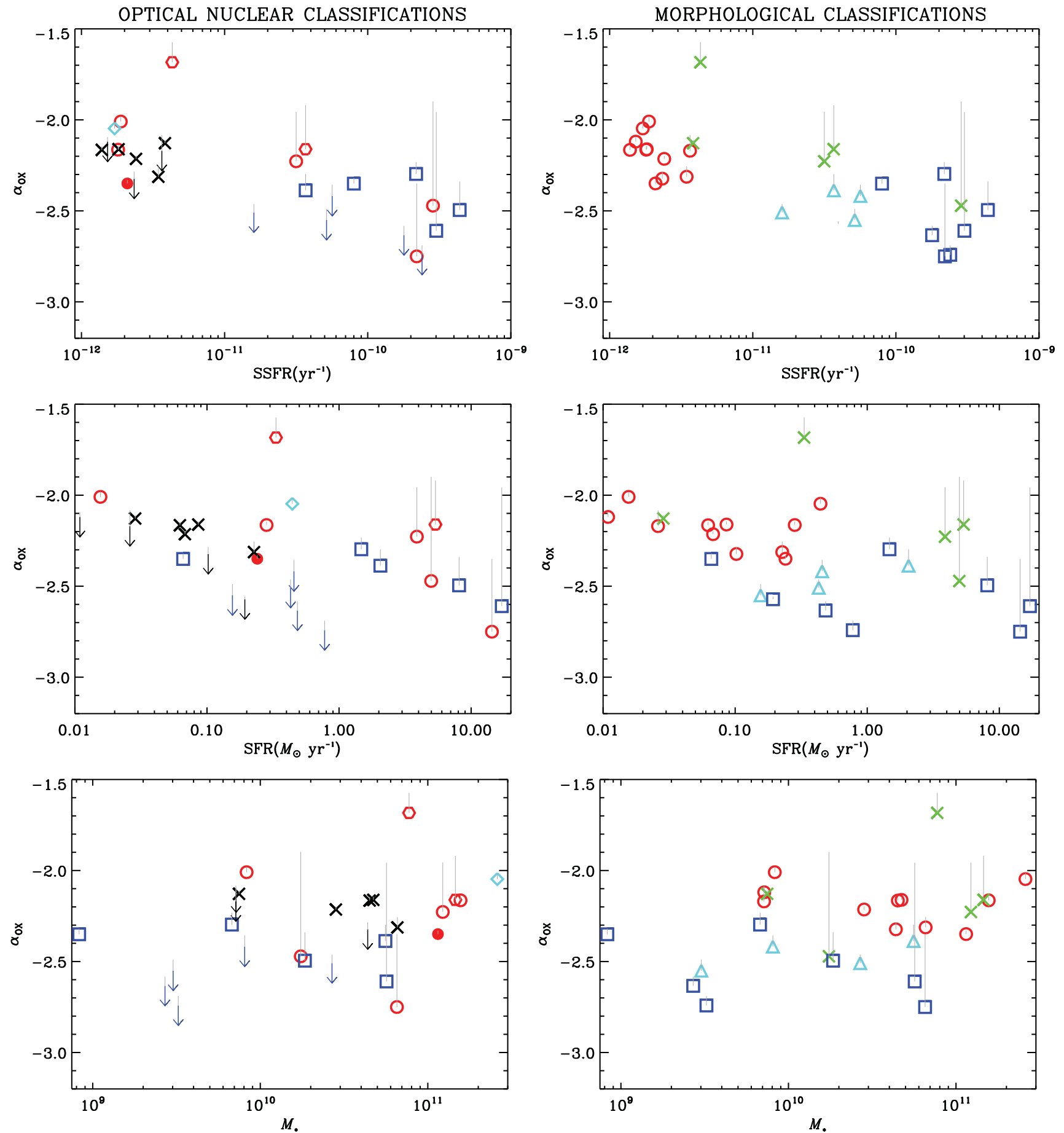

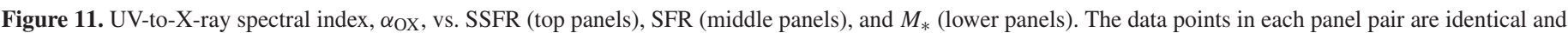

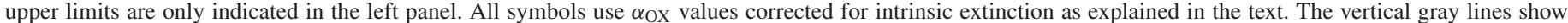

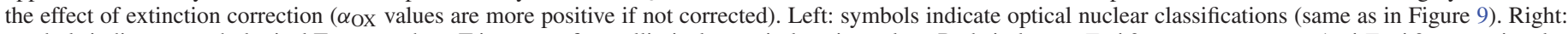

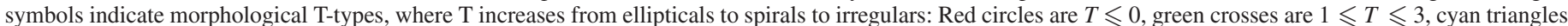

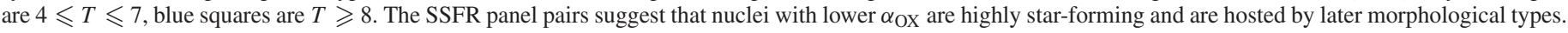
This trend is less pronounced in the SFR panels. The $M_{*}$ panels show that AGNs are hosted by the more massive systems and the earlier morphological types.

(A color version of this figure is available in the online journal.)

optical classification (marked by black crosses or downward pointing arrows in this panel) are most likely AGNs, LINERs, or transition objects.

On the other hand, symbols in the right panel indicate the RC3-based morphological classifications of the host galaxies. A value of $\alpha_{\mathrm{OX}} \sim-2.3$ here roughly separates ellipticals (with low SSFRs) from galaxies with progressively more spiral morphologies (and higher SSFRs). This is consistent with our prediction for unknown nuclear activity systems in the left panel, as these nuclei are hosted by morphologically earliertype systems.

Since SSFR is SFR normalized by stellar mass, $M_{*}$, it is interesting to also investigate possible trends of $\alpha_{\mathrm{OX}}$ with these quantities separately. In the middle and bottom rows of Figure 11 
we show pairs of plots for $\alpha_{\mathrm{OX}}$ versus SFR and $\alpha_{\mathrm{OX}}$ versus $M_{*}$. Although there is a trend for lower SFR (and higher $M_{*}$ ) systems to have larger values of $\alpha_{\mathrm{OX}}$, this is not as pronounced as the trend with SSFR.

\subsection{Multi-wavelength Classifications (Table 11)}

Table 11 brings together results on the type of activity of HCG nuclei from different wavelength ranges. The information presented includes X-ray -UV nuclear counterparts (columns 3 and 4, Section 5.3), UV-to-X-ray spectral indices (columns 5 and 6, Section 5.3), optical emission line ratio classifications (column 7, Section 5.1.1), level of X-ray nuclear activity (column 8, Section 3.1), radio nuclear excess (column 9, Section 5.2), and HST -based nuclear detections (columns 10 and 11, Section 5.1.2).

Considering this table as a whole, we can draw a number of useful conclusions.

1. For about $60 \%(22 / 37)$ of HCG galaxies we have detected a single, nuclear X-ray point source, as indicated by the overlap between the X-ray and UV PSFs in the central region of a galaxy ("y" in column 4). Two of these galaxies are classified as optAGN, one as opt-nonSF, one as optLINER/AGN, one as optLINER, two as optTO/LINER, two as optTO/SF, and three as optSF. Nine have no emission line ratios and are unclassified. Given Chandra's resolution, the detection of these nuclear X-ray sources is consistent with some level of AGN activity in these systems, although an XRB origin cannot be excluded (and could, in fact, dominate).

2. For $27 \%(10 / 37)$ of galaxies there is no detected nuclear X-ray emission at all ("n" in column 4). Five of these nuclei are classified optSF, four are unclassified and one is classified as optTO/LINER. This is HCG $90 \mathrm{D}$, which has a very irregular, clumpy appearance, likely related to its close interaction with 90 B. Although we formally identify the center of galaxies with a UV intensity peak, there is likely no well-defined nucleus for such a morphology. Depending on the slit position, it is unclear whether the optical spectroscopic classification corresponds to the same source.

3. For three galaxies $(\sim 8 \%)$, the identification of counterparts is questionable ("?" in column 4). Specifically, for HCG 31 Q, the Chandra PSF overlaps slightly with the UV PSF, but the source positions are also $\sim 2$.'3 apart, so the lack of coincidence is unlikely to be due to astrometric errors ("n?" in column 4). For HCG 59 B and 92 F, the X-ray sources are $\sim 2^{\prime \prime}$ from the UV intensity peak with little PSF overlap. These can only tentatively be considered as X-ray counterparts to the UV emission ("y?" in column 4).

4. As mentioned, in two cases $(\sim 5 \%)$ there are two possible X-ray counterparts for a single UV nuclear source. In particular, for HCG $31 \mathrm{ACE}$, the Chandra PSFs for X-ray sources 38 and 40 both overlap with the UV PSF. Since source 40 is only 0 .'7 away from the UV peak (cf. $1^{\prime \prime}$.7 for 38 ), we chose source 40 as the most likely counterpart. In the case of $22 \mathrm{~A}$ both X-ray PSFs are almost symmetrical about the UV peak, so not even a tentative choice is possible.

5. X-ray luminosity is often used as a direct diagnostic for the presence of an AGN, with $\sim 1 \times 10^{41-42} \mathrm{erg} \mathrm{s}^{-1}$ taken as a fiducial threshold for unambiguously strong AGN activity (e.g., Reynolds \& Nowak 2003; Bauer et al. 2004).
Using $L_{X, 0.5-8.0 \mathrm{keV}}=10^{41} \mathrm{erg} \mathrm{s}^{-1}$ as our threshold, we see that there are only three sources that are candidates for strong AGNs (column 8 in Table 11). These are HCGs 16 B, $90 \mathrm{~A}$, and $92 \mathrm{C}$. The first is classified as optLINER and the other two as optAGN. Thus, together with $62 \mathrm{~A}, \mathrm{HCG} 16 \mathrm{~B}$ is a second case where a LINER and an AGN may coexist.

6. According to column 7, there is one optLINER/AGN, one optAGN, and one opt-nonSF which, according to column 8, are not strong X-ray AGNs. Combining these results, we believe that these are good LLAGN candidates.

7. Columns 10 and 11 suggest that our HST data do not help us discriminate clearly between AGN and SF systems. For 22 sources the HST median-divided image suggests a nuclear detection ("y" in column 10). However, there is no one-to-one correspondence between these 22 sources and the 22 sources for which X-ray-UV counterparts exist. On the one hand, it is encouraging that for four of these, namely the nuclei of HCG 16 A, 22 A, 42 $\mathrm{A}$, and $92 \mathrm{C}$, which are classified as optLINER/AGN, optAGN, opt-nonSF, and optAGN, respectively (column 7), an optical nuclear source seems to be present in the median-divided image (column 10). However, combining this result with GALFIT is problematic for two main reasons. First, in general, GALFIT leads to fits that are poor and in most cases do not converge. This is because most of the galaxies in our sample are disturbed and thus a full Sersic profile fitting is usually unable to fit the diffuse light. Second, only one of the AGN candidate galaxies mentioned, HCG 42 A, has a GALFIT center that lies within the three-pixel positional uncertainty from the mediandivided center. For the other three galaxies, we are unable to obtain a satisfactory GALFIT fit. On the other hand, note that five galaxies whose optical classification is not AGN nevertheless have their GALFIT and median-divided centers within the three-pixel positional uncertainty. Given the lack of good GALFIT results, it is hard to assess the significance of this result.

\section{AGN FRACTION}

For the purpose of comparison with galaxy clusters, we calculate the AGN fraction in our compact group sample by following Martini et al. (2006). We estimate $3 \sigma$ uncertainties using the method of Gehrels (1986). We use $R$-band magnitudes from Hickson et al. (1989) to establish that in our sample there are 26 galaxies with $M_{R}<-20$. For four galaxies for which Hickson et al. (1989) do not provide $R$-band data we obtain magnitude information from the NASA/IPAC Extragalactic Database. We assume that the AGN hosts among these 26 galaxies are those that are optically identified as such (Column 7 in Table 11). This includes mixed (e.g., LINER/AGN) and tentative (AGN?) classifications. The inclusion of these latter cases does not actually matter, as we also use the further criterion that $L_{X, 0.5-8.0 \mathrm{keV}}>10^{41} \mathrm{erg} \mathrm{s}^{-1}$. This leaves only two galaxies, which are the two strongest AGN cases, namely HCG 90 A and HCG $92 \mathrm{C}$. The AGN fraction defined in this way for two out of 26 galaxies is then $f_{A}\left(M_{R}<-20, L_{X}>10^{41}\right)=0.08_{-0.01}^{+0.35}$. This is close, but higher than the $\sim 5 \%$ fraction of Martini et al. (2006) in galaxy clusters.

\section{DISCUSSION}

The main result of this paper is the significant detection of nuclear X-ray point sources in $60 \%$ of the $37 \mathrm{HCG}$ galaxies 
Table 12

Physical Size Estimate (kpc) of Areas Used for UV and X-Ray Nuclear Photometry

\begin{tabular}{lcc}
\hline \hline $\begin{array}{l}\text { Group ID } \\
(1)\end{array}$ & $\begin{array}{c}\text { Swift-UVOT }\left(10^{\prime \prime}\right) \\
(2)\end{array}$ & $\begin{array}{c}\text { Chandra }\left(1^{\prime \prime}\right) \\
(3)\end{array}$ \\
\hline HCG 7 & 2.7 & 0.3 \\
HCG 16 & 2.6 & 0.3 \\
HCG 22 & 1.8 & 0.2 \\
HCG 31 & 2.8 & 0.3 \\
HCG 42 & 3.0 & 0.3 \\
HCG 59 & 3.1 & 0.3 \\
HCG 62 & 3.1 & 0.3 \\
HCG 90 & 1.6 & 0.2 \\
HCG 92 & 4.3 & 0.4 \\
\hline
\end{tabular}

Notes. Columns are: 1: HCG group ID; 2: extent in kpc of $10^{\prime \prime}$ at the galaxy group distance. $10^{\prime \prime}$ is the diameter of the circular regions used for nuclear UVOT photometry. 3: Extent in kpc of $1^{\prime \prime}$, the on-axis Chandra PSF, at the galaxy group distance.

in this sample. We detect these sources by taking advantage of Chandra's excellent angular resolution, while also taking into account the size of the PSF at each source's detected position on the CCD and the overlap with the UV PSF.

Unfortunately, the mere detection of an X-ray point source that appears to coincide with the UV galactic nucleus is no strong evidence that the origin of the nuclear emission is an AGN rather than circumnuclear star formation, unless the source also has a high X-ray luminosity. The threshold we use in this paper is $L_{X, 0.5-8.0 \mathrm{keV}} \geqslant 10^{41} \mathrm{erg} \mathrm{s}^{-1}$ and most of our nuclear sources are fainter. We provide a quantitative illustration of this caveat in Table 12, where we give estimates of the physical sizes (in kiloparsecs) of the areas used for Chandra and UVOT photometry at the distances of our galaxies. Taking the case of Chandra, $1^{\prime \prime}$ at the distances of these galaxies corresponds to physical sizes ranging from $\sim 0.16$ (for $\mathrm{HCG} 90$, our nearest group) to $\sim 0.43 \mathrm{kpc}$ (for HCG 92). The known detections of nuclear star clusters with sizes of this order and ages as young as $10 \mathrm{Myr}$ demonstrates that regions of this physical size can be the sites of compact and intense circumnuclear star formation (e.g., Rossa et al. 2006) which, in turn, can harbor unresolved XRB populations. Since most of our nuclear sources have low $L_{X}$ estimates, such a possibility should be seriously considered. The optical emission-line ratio classifications for 22 galaxies in the sample are in support of the lack of strong AGN activity and the prevalence of star formation in HCG nuclei. We caution that these are small numbers, but, taken at face value, almost half of our systems (45.5\%) are classified as pure optSF, while more are classified as optTO or have mixed classifications. Only a minority (13.6\%) are optAGN, and two of these also have high $\mathrm{X}$-ray luminosities.

Two of the three nuclei that fulfill the X-ray luminosity criterion for harboring strong AGNs have $L_{X, 0.5-8.0 \mathrm{keV}}>$ $10^{42} \mathrm{erg} \mathrm{s}^{-1}$ (90 A and $92 \mathrm{C}$, both also optAGN). The result for HCG $90 \mathrm{~A}$ is also independently confirmed by LaMassa et al. (2011) who, using XMM-Newton data and detailed spectral fitting, report $L_{X, 2.0-10.0 \mathrm{keV}}=10^{42.96} \mathrm{erg} \mathrm{s}^{-1}$ (compare with $L_{X, 0.5-8.0 \mathrm{keV}}=10^{42.6} \mathrm{erg} \mathrm{s}^{-1}$ in our Table 4). These are the only nuclear sources in our sample that fulfill optical spectroscopic, X-ray luminosity, and, for $90 \mathrm{~A}$, radio excess criteria for unambiguous and strong AGNs.

As X-ray luminosity decreases, so does its discriminative power as an AGN diagnostic. Two nuclei have $10^{40.5}<$
$L_{X}<10^{42}$ (16A and 16B, optLINER/AGN and optLINER, respectively), but the optLINER/AGN is the X-ray fainter one. The rest of the systems are progressively fainter in the X-rays, precluding any conclusions on the nature of the nuclear activity from X-ray information alone. The conservative conclusion based on X-ray luminosity is to consider these as candidates either for nuclear star clusters and XRB hosts or LLAGN hosts (or, possibly, some combination of the two). Although X-ray faint $\left(L_{X, 2.0-10.0 \mathrm{keV}} \sim 10^{38} \mathrm{erg} \mathrm{s}^{-1}\right)$ nuclei have been identified as LLAGNs with Chandra data, e.g., in the Palomar sample of Ho et al. (2001), the comparison is not entirely fair as that sample contained early-type galaxies where star formation is unlikely to strongly dilute the AGN emission.

We have also combined X-ray and UV nuclear photometry to calculate $\alpha_{\text {OX }}$ values and compare with those for strong AGNs and star-forming galaxies. The KS test shows that HCG nuclei are completely distinct from strong AGNs and fairly similar, though not identical, to star-forming galaxies. The correlation in $\alpha_{\mathrm{OX}}-v L_{v 2600}$ space (Figure 9) for strong AGNs is well established over $\sim 4-6$ orders of magnitude (Strateva et al. 2005; Steffen et al. 2006; Just et al. 2007). Lusso et al. (2010) also confirm this correlation, slightly extending it to fainter systems. The location of HCG nuclei in Figure 9 is consistent with their $L_{X}$ values: the highest $L_{X}$ nuclei, i.e., those that are more likely to harbor strong AGNs, do, in fact, follow the correlation. For lower nuclear $L_{X}$ values, nuclei are located farther below the correlation. Notably, this region is also inhabited by the normal, star-forming galaxies of L10. Since this latter sample was compiled independently from our HCG sample, as well as the other comparison samples shown in Figure 9, this result is in support of our conclusion that strong AGN activity is absent and star formation may be dominant in most HCG nuclei.

Taking into account the optical AGN/TO/SF classification information, in Figure 9 there appears to be a broad transition region at $\alpha_{\mathrm{OX}} \sim-2.3$. At lower $\alpha_{\mathrm{OX}}$ values most of the nuclear emission is likely due exclusively to star-formation. This is consistent with the top row of Figure 11. The left panel shows that it is precisely the high SSFR systems that have $\alpha_{\mathrm{OX}} \lesssim-2.3$, and the right panel shows that these have the most spiral-like morphologies. In addition, the right panel shows a very smooth progression with morphological type toward values of $\alpha_{\mathrm{OX}} \lesssim$ -2.3 . Although we do not claim that $\alpha_{\mathrm{OX}} \gtrsim-2.3$ guarantees AGN activity, the fact that these systems are preferentially early-type (as well as the most massive, see bottom row of Figure 11) is consistent with earlier results that AGNs in compact groups are located preferentially in early type galaxies (Coziol et al. 1998b, 1998a). Note also that the trend with decreasing $\alpha_{\mathrm{OX}}$, although still present, is not as clear in the lower two panel rows of Figure 11. This simply reflects the fact that SSFR is by construction a better discriminator between highly star-forming late types and more quiescent (and more massive) early types.

One possible caveat in this work is the mismatch in resolution and, hence, photometric aperture between the X-ray and UV data. The use of a $10^{\prime \prime}$ diameter for UV photometry, corresponding to physical sizes between 1.6 and $4.3 \mathrm{kpc}$, means that some of the UV emission may not be co-spatial with emission from the X-ray source inside the smaller X-ray aperture. This would lead to an overestimate of the UV luminosity, and, hence, an underestimate of $\alpha_{\mathrm{OX}}$. In such a case, data points corresponding to the "true" $\alpha_{\text {OX }}$ and UV luminosity values in Figure 9 would be shifted to the left (toward lower $v L_{v 2600}$ 
values) and up (toward higher $\alpha_{\mathrm{OX}}$ ) relatively to the data points shown. Note, though, that, with the exception of possible nuclear star clusters, the UV aperture mostly traces galactic bulges, where old stellar populations dominate, and contamination from non-nuclear UV emission due to massive young stars might not be so important. Also, contamination from old stars (the socalled UV upturn) is not important at the near-UV wavelengths studied because it only becomes dominant blueward of $2000 \AA$ (O'Connell 1999). These observations are in line with the results of the tests by Grupe et al. (2010) who performed AGN UV photometry with Swift UVOT. They performed photometry both with the standard $5^{\prime \prime}$-radius UVOT aperture and a narrower $3^{\prime \prime}$-radius that included aperture corrections. They found that host galaxy contamination is most serious in the optical UVOT bands, while in the UV bands the magnitude difference was on the order of $0.05 \mathrm{mag}$. We should caution, however, that since Grupe et al. (2010) had bright AGNs, they were more likely to have the AGNs dominating both in the UV and the X-ray, which is more likely to drive their conclusions. Similarly, the $\alpha_{\mathrm{OX}}$ values of our known strong AGN systems do fall close to the strong AGN correlation. Finally, we test the effect of photometric aperture by performing X-ray photometry using the larger UV apertures. This leads, on average, to $L_{v, 2 \mathrm{keV}}$ values that are higher by $0.48 \mathrm{erg} \mathrm{s}^{-1} \mathrm{~Hz}^{-1}$ or $\alpha_{\text {OX }}$ values that increase by 0.18 . Such changes do not significantly affect the results of this work.

We are thus led to the conclusion that the observed trends of $\alpha_{\mathrm{OX}}$ with $\nu L_{v 2600}$ are telling us two things. First, HCG nuclei as a class generally do not harbor strong AGNs. Instead, the UV and X-ray emission we see is in most cases likely dominated by nuclear star formation. Second, considering early-and late-type morphologies, the most likely interpretation is that low SSFR early-type systems are the ones harboring weak AGNs, while high SSFR late-type systems are dominated by star formation.

The radio and $H S T$-based diagnostics do not provide much additional insight regarding the nuclear activity in this galaxy sample. For three galaxies, the possible detection of excess radio emission above that expected from star formation alone is consistent with the X-ray luminosity (for HCG $90 \mathrm{~A}$ ), as well as the optAGN classification (for HCG 16 A and 90 A). However, the excess is not greater than the uncertainty in the radio-SFR correlation used. We also have no radio data for any of the galaxies that lack optical classifications. Thus the radio results are not particularly useful.

Using HST-data for a subset of our galaxies, we detect what seem to be nuclear sources after subtracting mediansmoothed images for 22 galaxies. However, these sources do not correspond to the optical emission line ratio classifications in any systematic way. Due to the disturbed nature of most galaxies, we are also unable to obtain reliable and consistent surface brightness fits with GALFIT in most cases. The fact that some sources have their median divided center within 3 pixels of the GALFIT center is thus of limited significance.

Our AGN fraction result for compact groups suffers from small number statistics and needs to be tested with larger samples. However, taken at face value and given that we have applied the same criteria used by others to obtain the AGN fraction in galaxy clusters, our result is somewhat higher than the one for clusters. This might suggest that the lower velocity dispersions and shorter crossing times in compact groups, which make this environment physically distinct from that of clusters, also have an effect on AGN activity, just as they seem to for star formation.
We have no emission line ratios, and thus no optical spectroscopic classifications for 15 of our galaxies, indicated by black crosses and arrows in the figures. These systems include HCG 7B, 22B, 31F, 42B, 42C, 42D, 62B, 62C, 62D, 90B, 90C, $92 \mathrm{~B}, 92 \mathrm{D}$, and 92E. Based on the trends discussed above and the morphologies of these systems, we tentatively predict the nature of their nuclear activity. There are two late-type systems: HCG 31F (morphological type Im) and HCG 92B (type Sbc). Our prediction is that these will be dominated by star formation. The rest of the systems have elliptical/S0 morphologies and may harbor a weak LLAGN.

\section{CONCLUSIONS}

This paper presents the first compilation of X-ray detected point sources in the fields of 9 HCGs, for which we provide an extensive compilation of source characteristics.

We have used multi-wavelength diagnostics (X-ray, UV, optical, and radio) to assess the levels of AGN, SF, and LINER activity in the compact group environment. Our main results follow.

1. In $60 \%$ of 37 galaxies we detect single, nuclear X-ray sources that have nuclear UV counterparts. We detect no nuclear X-ray emission for $27 \%$ of our galaxies. The rest of the systems have more uncertain X-ray nuclear detections.

2. Out of the 22 galaxies for which emission line ratios are available in the literature, we classify a clear majority of them $(45.5 \%)$ as optSF. Our criteria allow us, for the first time, to also classify five systems as LINERs, although four of these are mixed (LINER/AGN, TO/LINER). Thus, any LINER activity is associated with a minority $(22.5 \%)$ of systems. Only three nuclei $(13.6 \%)$ are classified as optAGN.

3. Only three systems (HCG 16 B, 90 A, 92 C) are candidates for hosting an X-ray strong $\operatorname{AGN}\left(L_{X, 0.5-8.0 \mathrm{keV}} \geqslant\right.$ $10^{41} \mathrm{erg} \mathrm{s}^{-1}$ ).

4. When several criteria are taken into account (optical spectroscopic classification, excess radio emission, X-ray luminosity, location in $\alpha_{\mathrm{OX}}-v L_{v 2600}$ parameter space) only two HCG nuclei (90 A, 92 C) fulfill several criteria and are classified as strong unambiguous AGNs.

5. In $\alpha_{\mathrm{OX}}-v L_{v 2600}$ space, HCG nuclei occupy a region that is distinct from that occupied by strong AGNs and largely overlaps with that occupied by other nearby star-forming galaxies not known to harbor AGNs (Figure 9). The only exceptions are the two strong AGNs which do fall into the AGN region. We thus tentatively make the prediction that HCG nuclei without optical nuclear-type classifications are dominated by star formation (if they have late-type morphologies) or may harbor low luminosity AGNs (especially if they have early-type morphologies).

6. $\alpha_{\mathrm{OX}}$ anticorrelates with galaxy-wide SSFR and spiral morphology so that the star formation contribution is strongest in highest SSFR and later type morphology galaxies Figure 11. The detected anticorrelation (correlation) with $\operatorname{SFR}\left(M_{*}\right)$ is weaker.

7. Using the same criterion used in galaxy clusters (Martini et al. 2006), the AGN fraction of HCG galaxies more luminous than both $M_{R}=-20$ and $L_{X, 0.5-8.0 \mathrm{keV}}=$ $10^{41} \mathrm{erg} \mathrm{s}^{-1}$ is $0.08_{-0.01}^{+0.35}$, which is close to but higher than that in clusters.

Our general conclusion is that overall the CG environment has a mitigating effect on the level of AGN activity but not 
AGN numbers. With future expanded X-ray and UV samples as well as deeper observations we will be able to better assess the nature and statistics of HCG nuclei. The comparison with galaxy clusters suggests that environment plays a key role for the overall level of AGN activity. In this respect, it is imperative to carry out detailed comparisons with samples from other group, cluster, and field environments.

We gratefully acknowledge the support of the ACIS Instrument Team contract SV4-74018 (PI: G. P. Garmire). P.T. acknowledges support through a NASA Postdoctoral Program Fellowship at NASA Goddard Space Flight Center, administered by Oak Ridge Associated Universities through a contract with NASA. A.H. and P.T. were also supported by NASA ADAP 09-ADP09-0071 (PI: Hornschemeier). We thank Kip Kuntz for discussions and assistance with matching of sources in three $\mathrm{X}$-ray bands. We thank Bret Lehmer for making his catalog of star-forming galaxies available to us. K.F., T.D.D., and S.C.G thank the Natural Science and Engineering Research Council of Canada and the Ontario Early Researcher Award Program for support. W.N.B. acknowledges support from NASA ADP grant NNX10AC99G. J.C.C. and C.G. acknowledge funding that was provided through Chandra Award No. GO8-9124B issued by the Chandra X-Ray Observatory Center, which is operated by the Smithsonian Astrophysical Observatory under NASA contract NAS8-03060, grant number HST-GO-10787.15-A from the Space Telescope Science Institute which is operated by AURA, Inc., under NASA contract NAS 5-26555, and by the National Science Foundation under award 090894. The Institute for Gravitation and the Cosmos is supported by the Eberly College of Science and the Office of the Senior Vice President for Research at the Pennsylvania State University. K.F. acknowledges support from the Queen Elizabeth II Graduate Scholarships in Science and Technology. This research has made use of data obtained from the High Energy Astrophysics Science Archive Research Center (HEASARC), provided by NASA's Goddard Space Flight Center. This research has made use of the NASA/IPAC Extragalactic Database (NED) which is operated by the Jet Propulsion Laboratory, California Institute of Technology, under contract with the National Aeronautics and Space Administration.

Facilities: Chandra, Swift

\section{REFERENCES}

Arnaud, K. A. 1996, in ASP Conf. Ser. 101, Astronomical Data Analysis Software and Systems V, ed. G. H. Jacoby \& J. Barnes (San Francisco, CA: ASP), 17

Baldwin, A., Phillips, M. M., \& Terlevich, R. 1981, PASP, 93, 817

Barth, A. J., Ho, L. C., Filippenko, A. V., \& Sargent, W. L. W. 1998, ApJ, 496, 133

Bauer, F. E., Alexander, D. M., Brandt, W. N., et al. 2004, AJ, 128, 2048

Becker, R. H., White, R. L., \& Helfand, D. J. 1995, ApJ, 450, 559

Bell, E. F. 2003, ApJ, 586, 794

Bock, D. C.-J., Large, M. I., \& Sadler, E. M. 1999, AJ, 117, 1578

Breeveld, A. A., Curran, P. A., Hoversten, E. A., et al. 2010, MNRAS, 406, 1687

Broos, P. S., Townsley, L. K., Feigelson, E. D., et al. 2010, ApJ, 714, 1582

Cappelluti, N., Hasinger, G., Brusa, M., et al. 2007, ApJS, 172, 341

Cardelli, J. A., Clayton, G. C., \& Mathis, J. S. 1989, ApJ, 345, 245

Cisternas, M., Jahnke, K., Inskip, K. J., et al. 2011, ApJ, 726, 57

Condon, J. J., Cotton, W. D., Greisen, E. W., et al. 1998, AJ, 115, 1693

Coziol, R., Brinks, E., \& Bravo-Alfaro, H. 2004, AJ, 128, 68

Coziol, R., de Carvalho, R. R., Capelato, H. V., \& Ribeiro, A. L. B. 1998a, ApJ, 506, 545

Coziol, R., Ribeiro, A. L. B., de Carvalho, R. R., \& Capelato, H. V. 1998b, ApJ, 493,563
De Breuck, C., Tang, Y., de Bruyn, A. G., Röttgering, H., \& van Breugel, W. 2002, A\&A, 394, 59

Deng, X.-F., Yu, G., \& Jiang, P. 2013, PASA, 30, 18

Desjardins, T. D., Gallagher, S. C., Tzanavaris, P., et al. 2013, ApJ, 763, 121

Dopita, M. A., Koratkar, A. P., Evans, I. N., et al. 1996, in ASP Conf. Ser. 103,

The Physics of Liners in View of Recent Observations, ed. M. Eracleous, A. Koratkar, C. Leitherer, \& L. Ho (San Francisco, CA: ASP), 44

Dressler, A., Thompson, I. B., \& Shectman, S. A. 1985, ApJ, 288, 481

Ehlert, S., Allen, S. W., Brandt, W. N., et al. 2013, MNRAS, 428, 3509

Eracleous, M., Hwang, J. A., \& Flohic, H. M. L. G. 2010a, ApJ, 711, 796

Eracleous, M., Hwang, J. A., \& Flohic, H. M. L. G. 2010b, ApJS, 187, 135

Fedotov, K., Gallagher, S. C., Konstantopoulos, I. S., et al. 2011, AJ, 142, 42

Ferland, G. J., \& Netzer, H. 1983, ApJ, 264, 105

Filho, M. E., Fraternali, F., Markoff, S., et al. 2004, A\&A, 418, 429

Filippenko, A. V., \& Terlevich, R. 1992, ApJL, 397, L79

Flohic, H. M. L. G., Eracleous, M., Chartas, G., Shields, J. C., \& Moran, E. C. 2006, ApJ, 647, 140

Freeman, P. E., Kashyap, V., Rosner, R., \& Lamb, D. Q. 2002, ApJS, 138, 185 Fukugita, M., Shimasaku, K., \& Ichikawa, T. 1995, PASP, 107, 945

Gallagher, S. C., Durrell, P. R., Elmegreen, D. M., et al. 2010, AJ, 139, 545

Gallagher, S. C., Johnson, K. E., Hornschemeier, A. E., Charlton, J. C., \& Hibbard, J. E. 2008, ApJ, 673, 730

Gallagher, S. C., Richards, G. T., Hall, P. B., et al. 2005, AJ, 129, 567

Gehrels, N. 1986, ApJ, 303, 336

Gehrels, N., Chincarini, G., Giommi, P., et al. 2004, ApJ, 611, 1005

Georgakakis, A., Coil, A. L., Laird, E. S., et al. 2009, MNRAS, 397, 623

González-Martín, O., Masegosa, J., Márquez, I., Guainazzi, M., \& JiménezBailón, E. 2009, A\&A, 506, 1107

Grogin, N. A., Conselice, C. J., Chatzichristou, E., et al. 2005, ApJL, 627, L97 Grupe, D., Komossa, S., Leighly, K. M., \& Page, K. L. 2010, ApJS, 187, 64

Halpern, J. P., \& Steiner, J. E. 1983, ApJL, 269, L37

Heckman, T. M. 1980, A\&A, 87, 152

Hickox, R. C., \& Markevitch, M. 2006, ApJ, 645, 95

Hickson, P. 1982, ApJ, 259, 930

Hickson, P., Kindl, E., \& Auman, J. R. 1989, ApJS, 70, 687

Hickson, P., Mendes de Oliveira, C., Huchra, J. P., \& Palumbo, G. G. 1992, ApJ, 399,353

Ho, L. C., Feigelson, E. D., Townsley, L. K., et al. 2001, ApJL, 549, L51

Ho, L. C., Filippenko, A. V., \& Sargent, W. L. W. 1997, ApJ, 487, 568

Hopkins, P. F., \& Quataert, E. 2010, MNRAS, 407, 1529

Jeltema, T. E., Binder, B., \& Mulchaey, J. S. 2008, ApJ, 679, 1162

Johnson, K. E., Hibbard, J. E., Gallagher, S. C., et al. 2007, AJ, 134, 1522

Just, D. W., Brandt, W. N., Shemmer, O., et al. 2007, ApJ, 665, 1004

Kartaltepe, J. S., Sanders, D. B., Le Floc'h, E., et al. 2010, ApJ, 721, 98

Kashyap, V. L., van Dyk, D. A., Connors, A., et al. 2010, ApJ, 719, 900

Kauffmann, G., Heckman, T. M., Tremonti, C., et al. 2003, MNRAS, 346,1055

Kennicutt, R. C. 1998, ARA\&A, 36, 189

Kewley, L. J., Dopita, M. A., Sutherland, R. S., Heisler, C. A., \& Trevena, J. 2001, ApJ, 556, 121

Kewley, L. J., Groves, B., Kauffmann, G., \& Heckman, T. 2006, MNRAS, 372,961

Kim, D.-W., Cameron, R. A., Drake, J. J., et al. 2004, ApJS, 150, 19

Konstantopoulos, I. S., Gallagher, S. C., Fedotov, K., et al. 2010, ApJ, 723, 197

Kraft, R. P., Burrows, D. N., \& Nousek, J. A. 1991, ApJ, 374, 344

LaMassa, S. M., Heckman, T. M., Ptak, A., et al. 2011, ApJ, 729, 52

Lee, B. C., Allam, S. S., Tucker, D. L., et al. 2004, AJ, 127, 1811

Lehmer, B. D., Alexander, D. M., Bauer, F. E., et al. 2010, ApJ, 724, 559

Lusso, E., Comastri, A., Vignali, C., et al. 2010, A\&A, 512, A34

Ma, C., \& Feissel, M. 1998, yCat, 1251, 0

Mamon, G. A. 1994, in Clusters of Galaxies, ed. F. Durret, A. Mazure, \& J. Tran Thanh Van (Singapore: Editions Frontieres), 291

Maoz, D., Nagar, N. M., Falcke, H., \& Wilson, A. S. 2005, ApJ, 625, 699

Martínez, M. A., Del Olmo, A., Coziol, R., \& Perea, J. 2010, AJ, 139, 1199

Martini, P., Kelson, D. D., Kim, E., Mulchaey, J. S., \& Athey, A. A. 2006, ApJ, 644,116

Mihos, J. C., \& Hernquist, L. 1996, ApJ, 464, 641

Mukai, K. 1993, Legacy, 3, 21

Mulchaey, J. S., Davis, D. S., Mushotzky, R. F., \& Burstein, D. 2003, ApJS, 145,39

Nagar, N. M., Falcke, H., \& Wilson, A. S. 2005, A\&A, 435, 521

O'Connell, R. W. 1999, ARA\&A, 37, 603

O'Sullivan, E., Giacintucci, S., Vrtilek, J. M., Raychaudhury, S., \& David, L. P. 2009, ApJ, 701, 1560

Peng, C. Y., Ho, L. C., Impey, C. D., \& Rix, H.-W. 2010, AJ, 139, 2097

Ponman, T. J., Bourner, P. D. J., Ebeling, H., \& Böhringer, H. 1996, MNRAS, 283, 690 
Poole, T. S., Breeveld, A. A., Page, M. J., et al. 2008, MNRAS, 383, 627

Rafferty, D. A., Bîrzan, L., Nulsen, P. E. J., et al. 2013, MNRAS, 428, 58

Ranalli, P., Comastri, A., \& Setti, G. 2003, A\&A, 399, 39

Ranalli, P., Comastri, A., Zamorani, G., et al. 2012, A\&A, 542, A16

Rasmussen, J., Ponman, T. J., Verdes-Montenegro, L., Yun, M. S., \& Borthakur, S. 2008, MNRAS, 388, 1245

Reynolds, C. S., \& Nowak, M. A. 2003, PhR, 377, 389

Ribeiro, A. L. B., de Carvalho, R. R., Capelato, H. V., \& Zepf, S. E. 1998, ApJ, 497, 72

Roche, P. F., Aitken, D. K., Smith, C. H., \& Ward, M. J. 1991, MNRAS, 248, 606

Roming, P. W. A., Kennedy, T. E., Mason, K. O., et al. 2005, SSRv, 120,95

Rossa, J., van der Marel, R. P., Böker, T., et al. 2006, AJ, 132, 1074

Schlegel, D. J., Finkbeiner, D. P., \& Davis, M. 1998, ApJ, 500, 525

Shen, Y., Mulchaey, J. S., Raychaudhury, S., Rasmussen, J., \& Ponman, T. J. 2007, ApJL, 654, L115

Shields, J. C. 1992, ApJL, 399, L27

Silverman, J. D., Kampczyk, P., Jahnke, K., et al. 2011, ApJ, 743, 2
Smith, B. J., Swartz, D. A., Miller, O., et al. 2012, AJ, 143, 144

Stasińska, G., Cid Fernandes, R., Mateus, A., Sodré, L., \& Asari, N. V. 2006, MNRAS, 371, 972

Steffen, A. T., Brandt, W. N., Alexander, D. M., Gallagher, S. C., \& Lehmer, B. D. 2007, ApJL, 667, L25

Steffen, A. T., Strateva, I., Brandt, W. N., et al. 2006, AJ, 131, 2826

Strateva, I. V., Brandt, W. N., Schneider, D. P., Vanden Berk, D. G., \& Vignali, C. 2005, AJ, 130, 387

Tananbaum, H., Avni, Y., Branduardi, G., et al. 1979, ApJL, 234, L9

Terlevich, R., \& Melnick, J. 1985, MNRAS, 213, 841

Tzanavaris, P., Hornschemeier, A. E., Gallagher, S. C., et al. 2010, ApJ, 716,556

Veilleux, S., \& Osterbrock, D. E. 1987, ApJS, 63, 295

Verdes-Montenegro, L., Yun, M. S., Williams, B. A., et al. 2001, A\&A, 377, 812

Walker, L. M., Johnson, K. E., Gallagher, S. C., et al. 2010, AJ, 140, 1254

Walker, L. M., Johnson, K. E., Gallagher, S. C., et al. 2012, AJ, 143, 69

Xu, C. K., Iglesias-Páramo, J., Burgarella, D., et al. 2005, ApJL, 619, L95

Xue, Y. Q., Luo, B., Brandt, W. N., et al. 2011, ApJS, 195, 10 